\title{
Polygenic risk modeling for prediction of epithelial ovarian
} \section{cancer risk}

\author{
(c) The Author(s) 2021
}

Polygenic risk scores (PRS) for epithelial ovarian cancer (EOC) have the potential to improve risk stratification. Joint estimation of Single Nucleotide Polymorphism (SNP) effects in models could improve predictive performance over standard approaches of PRS construction. Here, we implemented computationally efficient, penalized, logistic regression models (lasso, elastic net, stepwise) to individual level genotype data and a Bayesian framework with continuous shrinkage, "select and shrink for summary statistics" (S4), to summary level data for epithelial non-mucinous ovarian cancer risk prediction. We developed the models in a dataset consisting of 23,564 non-mucinous EOC cases and 40,138 controls participating in the Ovarian Cancer Association Consortium (OCAC) and validated the best models in three populations of different ancestries: prospective data from 198,101 women of European ancestries; 7,669 women of East Asian ancestries; 1,072 women of African ancestries, and in 18,915 BRCA1 and 12,337 BRCA2 pathogenic variant carriers of European ancestries. In the external validation data, the model with the strongest association for nonmucinous EOC risk derived from the OCAC model development data was the S4 model (27,240 SNPs) with odds ratios (OR) of 1.38 (95\% Cl: 1.28-1.48, AUC: 0.588) per unit standard deviation, in women of European ancestries; 1.14 (95\% Cl: 1.08-1.19, AUC: 0.538) in women of East Asian ancestries; 1.38 (95\% Cl: 1.21-1.58, AUC: 0.593) in women of African ancestries; hazard ratios of 1.36 (95\% Cl: 1.29-1.43, AUC: 0.592) in BRCA1 pathogenic variant carriers and 1.49 (95\% Cl: 1.35-1.64, AUC: 0.624) in BRCA2 pathogenic variant carriers. Incorporation of the S4 PRS in risk prediction models for ovarian cancer may have clinical utility in ovarian cancer prevention programs.

European Journal of Human Genetics (2022) 30:349-362; https://doi.org/10.1038/s41431-021-00987-7

\section{INTRODUCTION}

Rare variants in known high and moderate penetrance susceptibility genes (BRCA1, BRCA2, BRIP1, PALB2, RAD51C, RAD51D and the mis-match repair genes) account for about $40 \%$ of the inherited component of EOC disease risk [1, 2]. Common susceptibility variants, reviewed in Kar et al. and Jones et al., explain about $6 \%$ of the heritability of EOC $[1,3]$. Polygenic risk scores (PRS) provide an opportunity for refined risk stratification in the general population and in carriers of rare moderate or high risk alleles.

A PRS is calculated as the weighted sum of the number of risk alleles carried for a specified set of variants. The best approach to identify the variant set and their weights to optimize the predictive power of a PRS is unknown. A common approach involves selecting a set of variants that reach a threshold for association based on the $p$-value for each variant with or without pruning to remove highly correlated variants $[4,5]$. More complex machine learning approaches that do not assume variant independence have also been used [6,7], but these methods have produced only modest gains in predictive power for highly polygenic phenotypes [6, 8]. Penalized regression approaches such as the lasso, elastic net and the adaptive lasso have also been used with individual level data [9], but a major drawback is the computational burden required to fit the models $[9,10]$.

We present novel, computationally efficient PRS models using two approaches: (1) penalized regression models including the lasso, elastic net and minimax concave penalty (MCP) for use with individual genotype data; and (2) a Bayesian regression model with continuous shrinkage priors for use where only summary statistics are available-referred to as the "select and shrink with summary statistics" (S4) method. We compare these models with two commonly used methods, stepwise regression with $p$-value thresholding and LDPred.

\section{MATERIALS (SUBJECTS) AND METHODS Model development study population}

EOC is a highly heterogeneous phenotype with five major histotypes for invasive disease-high-grade serous, low-grade serous, endometrioid, clear cell, and mucinous histotype. The mucinous histotype is the least common and its origin is the most controversial with up to $60 \%$ of diagnosed cases of mucinous ovarian cancer often being misdiagnosed metastasis from nonovarian sites [11]. Therefore, in this study, we performed PRS modeling and association testing for all cases of invasive, nonmucinous EOC. We used genotype data from 23,564 invasive nonmucinous EOC cases and 40,138 controls with $>80 \%$ European ancestries from 63 case-control studies included in the Ovarian Cancer Association Consortium (OCAC) for model development. The distribution of cases by histotype was high-grade serous $(13,609)$, low-grade serous $(2,749)$, endometrioid $(2,877)$, clear cell $(1,427)$, and others $(2,902)$. Sample collection, genotyping, and

A full list of authors and their affiliations appears at the end of the paper 
quality control have been previously described [12]. Genotype data were imputed to the Haplotype Reference Consortium reference panel using 470,825 SNPs that passed quality control. Of the 32 million SNPs imputed, 10 million had imputation $r^{2}>0.3$ and were included in this analysis.

\section{Model validation study populations}

We validated the best-fitting PRS models developed in the OCAC data in 657 prevalent and incident cases of invasive, nonmucinous EOC and 198,101 female controls of European ancestries from the UK Biobank. Samples were genotyped using either the Affymetrix UK BiLEVE Axiom Array or Affymetrix UK Biobank Axiom Array (which share 95\% marker content), and then imputed to a combination of the Haplotype Reference Consortium, the 1000 Genomes phase 3 and the UK10K reference panels [13]. We restricted analysis to genetically confirmed females of European ancestries. We excluded individuals if they were outliers for heterozygosity, had low genotyping call rate $<95 \%$, had sex chromosome aneuploidy, or if they were duplicates (cryptic or intended) [12]. All SNPs selected in the model development phase were available in the UK Biobank.

We investigated transferability of the best-fitting PRS models to populations of non-European ancestries using genotype data from females of East Asian and African ancestries genotyped as part of the OCAC OncoArray Project [14, 15]. Women of East Asian ancestries-2,841 non-mucinous invasive EOC and 4,828 controls -were identified using a criterion of $>80 \%$ Asian ancestries. This included samples collected from studies in China, Japan, Korea, and Malaysia as well as samples collected from women of Asian ancestry in studies conducted in the US, Europe and Australia [14]. Similarly, women of African ancestries-368 cases of nonmucinous invasive EOC and 704 controls-mainly from studies conducted in the US, were identified using a criterion of $>80 \%$ African ancestries as described previously [15].

We also assessed the performance of the best-fitting PRS models in women of European ancestries ( $>80 \%$ European ancestries) with the pathogenic $B R C A 1$ and $B R C A 2$ variants from the Consortium of Investigators of Modifiers of BRCA1/2 (CIMBA). We used genotype data from 18,915 BRCA1 (2,053 invasive EOC cases) and 12,337 BRCA2 (717 invasive EOC cases) pathogenic variant carriers from 63 studies contributing to CIMBA [16]. Genotyping, data quality control measures, intercontinental ancestries assessment and imputation to the HRC reference panel are as described for the OCAC study population.

\section{STATISTICAL ANALYSIS}

\section{Polygenic risk models}

For all PRS models, we created scores as linear functions of the allele dosage in the general form $P R S_{i}=\sum_{j}^{p} x_{i j} \beta_{j}$ where genotypes are denoted as $x$ (taking on the minor allele dosages of 0,1 , and 2 ), with $x_{i j}$ representing the ith individual for the jth SNP (out of $p$ SNPs) on an additive log scale and $\beta_{j}$ represents the weight-the log of the odds ratio- of the jth SNP. We used different approaches to select and derive the optimal weights, $\beta_{j}$, in models as described below.
Penalized Regression Models

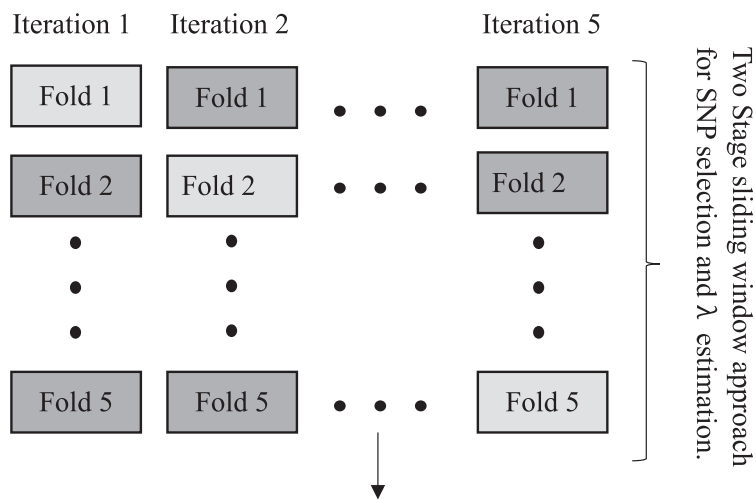

Select penalty function based on maximum mean AUC in 5fold validation for lasso, elastic net, MCP and hard threshold models

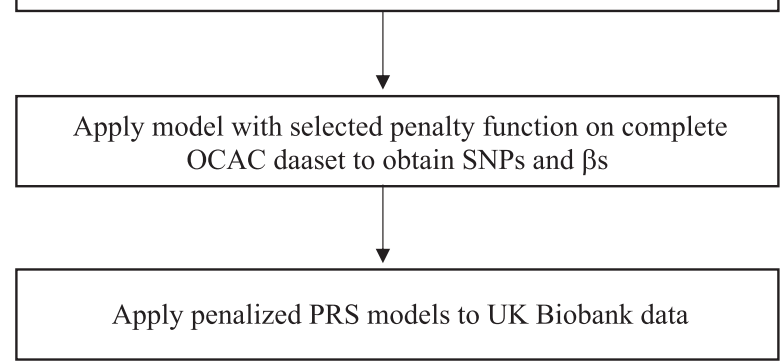

Apply penalized PRS models to UK Biobank data

Test
data $\quad \begin{gathered}\text { Training } \\ \text { data }\end{gathered}$

LDPred Models

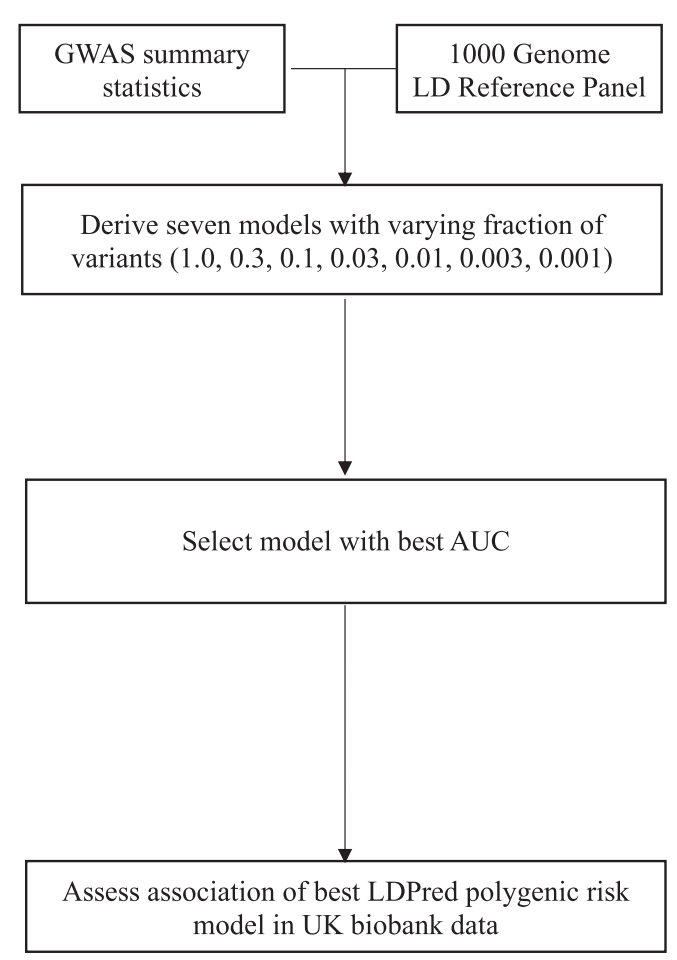

Fig. 1 PRS model development using penalized regression and LDPred Bayesian approach. Shown in the left panel is the two-stage approach with five-fold cross validation used for individual level genotype data while the right panel shows the LDPred approach used for summary level data. 
Penalized logistic regression models

A penalized logistic regression model for a set of SNPs aims to identify a set of regression coefficients that minimize the regularized loss function given by

$\operatorname{plr}(x ; \lambda, \kappa)=\left\{\begin{array}{c}x-\lambda \operatorname{sign}(x) /(1-\kappa) \text { if }|x|<\lambda / \kappa \text { and }|(x)|>\lambda \\ x \text { if }|x| \geq \frac{\lambda}{\kappa} \\ 0 \text { if }|(x)|<\lambda\end{array}\right.$

where $x$ is the effect estimate of a SNP, $\lambda$ is the tuning parameter and $K$ is the threshold (penalty) for different regularization paths. $\lambda$ and $K$ are parameters that need to be chosen during model development to optimize performance. The lasso, elastic net, MCP, and $p$-value thresholds are instances of the function with different $\kappa$ values. We minimized the winner's curse effect on inflated effect estimates for rare SNPs by penalizing rarer SNPs more heavily than common SNPs. Details are provided in the Supplementary Methods.

We used a two-stage approach to reduce computational burden without a corresponding loss in predictive power. The first stage was a SNP selection stage using a sliding windows approach, with $5.5 \mathrm{Mb}$ data blocks and a $500 \mathrm{~kb}$ overlap between blocks. SNP selection was performed for each block and selected SNPs were collated. Single SNP association analyses were then run, and all SNPs with a $x^{2}$ test statistic of less than 2.25 were excluded. The 2.25 cutoff was arbitrary and selected to maximize computational efficiency without loss in predictive power. Penalized regression models were applied to the remaining SNPs using $\lambda$ values of 3.0 and $k$ values of $0.0,0.2,0.4,0.6$, 0.8 and 1.0. SNPs selected in any of these models were included in subsequent analyses. In the second stage, we fit penalized regression models to the training dataset with $\lambda$ values ranging from 3.0 to 5.5 in increments of 0.1 iterated over $\kappa$ values from -3.0 to 1 in increments of 0.1 . The lasso model $(k=0)$ for each value of $\lambda$ was fitted first, to obtain a unique maximum. From the fitted maximum the $\kappa$ value was changed, and the model refitted.

We applied this two-stage approach with five-fold cross-validation (Fig. 1). In each iteration, the data set was split into five, with one part constituting the test data and the other four constituting the training data. The variants and their weights from the two-stage penalized logistic regression modeling in the training data were used to calculate the area under the receiver operating characteristic curve (AUC) in the test data in each iteration. AUC estimates for each combination of $\lambda$ and $\kappa$ were obtained. We repeated this process for each cross-validation iteration to obtain a mean AUC for each combination of $\lambda$ and $\kappa$. Finally, we selected the tuning and threshold parameters from the lasso, elastic net and MCP models with the maximum mean cross-validated AUC and fitted penalized logistic regression models with these parameters to the entire OCAC dataset to obtain SNP weights for PRS scores.

\section{Stepwise logistic regression with variable $\boldsymbol{P}$-value threshold}

This model is a general PLR model with $K=1$. As with the other PLR models, we investigated various values for $\lambda$ values (corresponding to a variable $P$-value threshold for including a SNP in the model). However, we observed that the implementation of this model on individual level data was more difficult than for other $K$ values because the model would sometimes converge to a local optimum rather than the global optimum. Therefore, we applied an approximate conditional and joint association analysis using summary level statistics correcting for estimated LD between SNPs, and utilizing a reference panel of 5,000 individual level genotype OCAC data as described in Yang et al. [17]. Details are provided in the Supplementary Methods.

\section{LDPred}

LDPred is a Bayesian approach that shrinks the posterior mean effect size of each marker based on a point-normal prior and LD information from an external reference panel. We derived seven candidate PRSs assuming the fractions of associated variants were $0.001,0.003,0.01,0.03,0.1,0.3$, and 1.0 respectively using the default parameters as detailed in Vilhjálmsson et al. [18] and an LD reference panel of 503 samples of European ancestries from the 1000 Genomes phase 3 release with effect estimates from the OCAC model development data.

\section{Select and shrink using summary statistics (S4)}

The S4 algorithm is similar to the PRS-CS algorithm [19]-a Bayesian method that uses summary statistics and between-SNP correlation data from a reference panel to generate the PRS scores by placing a continuous shrinkage prior on effect sizes. We adapted this method with penalization of rarer SNPs by correcting for the standard deviation resulting in the selection of fewer SNPs. We varied three parameters, $a, b, \varphi$, which control the degree of shrinkage of effect estimates. $\Phi$, the overall shrinkage parameter, is influenced by values of $a$ which controls shrinkage of effect estimates around 0 and $b$ which control shrinkage of larger effect estimates. We generated summary statistics for each crossvalidation training set and selected the parameters that gave the best results on average from the cross-validation and applied these to the set of summary statistics for the complete OCAC data set to obtain the final set of weights.

\section{PRS based on meta-analysis of OCAC-CIMBA summary statistics}

We conducted a meta-analysis of the EOC associations in BRCA1 variant carriers, $B R C A 2$ variant carriers and the participants participating in OCAC (see Supplementary Methods) and constructed two PRS models. An S4 PRS was generated by applying the $a, b$ and $\varphi$ parameters from the S4 model described above. A stepwise PRS was generated by selecting all SNPs that were genome-wide significant $\left(p<5 \times 10^{-8}\right)$ in the meta-analysis, along with any independent signals in the same region with $p<10^{-5}$ from the histotype specific analyses for low-grade serous, highgrade serous, endometrioid, clear cell ovarian cancer and nonmucinous invasive EOC.

\section{Polygenic risk score performance}

The best lasso, elastic net, stepwise and S4 models from the model development stage were validated using two independent data sources: the UK Biobank data and BRCA1/BRCA2 pathogenic variant carriers from the CIMBA. In the UK Biobank data, we evaluated discriminatory performance of the models using the AUC and examined the association between standardized PRS and risk of non-mucinous EOC using logistic regression analysis. For the CIMBA data, we assessed associations for each version of the PRS and invasive non-mucinous EOC risk using weighted Cox regression methods [20]. PRSs in the CIMBA data were scaled to the same PRS standard deviations as the OCAC data, meaning that per standard deviation hazard ratios estimated on CIMBA data are comparable to PRS associations in the OCAC and UK Biobank data. The regression models were adjusted for birth cohort $(<1920$, 1920-1929, 1930-1939, 1940-1949, 21950) and the first four ancestries informative principal components (calculated separately by iCOGS/OncoArray genotyping array) and stratified by Ashkenazi Jewish ancestries and country. Absolute risks by PRS percentiles adjusting for competing risks of mortality from other causes were calculated as described in the Supplementary Material.

\section{Transferability of PRS scores to non-European ancestries} We implemented two straightforward approaches to disentangle the role of ancestries on polygenic risk scoring. We selected homogenous ancestral samples by using a high cut-off criterion of $80 \%$ ancestries and we standardized the PRSs by mean-centering within each population. These approaches led to a more uniform 
distribution of PRSs within each ancestral population. Further adjustments using principal components of ancestries did not attenuate risk estimates.

\section{RESULTS}

\section{Model development}

The results for the models based on individual level genotype data are shown in Table 1. The elastic net model had the best predictive accuracy (AUC $=0.586$ ). The optimal value of $\lambda$ obtained from regularization paths for the MCP model was 3.3 meaning the best MCP model was equivalent to the lasso model. The best-fitting model based on summary statistics was the S4 (AUC $=0.593$ ) and the LDPred model had the poorest performance of the methods tested (AUC $=0.552$ ). Therefore, the LDPred model was not considered for further validation in other datasets. All SNPs selected and the associated weights for each model are provided in Supplementary Tables 1-6.

\section{Model validation in women of European ancestries}

Overall the PLR models performed slightly better in the UK Biobank data than the model development data (Table 2). Of the models developed using the OCAC model development data, the association was strongest with the S4 PRS. In BRCA1 and BRCA2 variant carriers, prediction accuracy was generally higher among $B R C A 2$ carriers than BRCA1 carriers. Consistent with results from the general population in the UK Biobank, the S4 PRS model also had the strongest association and predictive accuracy for invasive
EOC risk in both $B R C A 1$ and $B R C A 2$ carriers. Sensitivity analyses were conducted in which the unadjusted models for $B R C A 1$ and $B R C A 2$ carriers were progressively adjusted for birth cohort and 6 principal components. There was little difference in HR estimates and association $P$-values going from the unadjusted model to the model adjusting for six principal components (Supplementary Table 7). The PRS models developed using the OCAC-CIMBA metaanalysis results had better discriminative ability in the UK Biobank than the PRS models developed using only OCAC data. Compared with the S4 PRS using only OCAC data, the S4 PRS model derived from the meta-analysis had fewer SNPs, a stronger association with invasive EOC risk and better predictive accuracy. Similarly, the stepwise model from the OCAC-CIMBA meta-analysis performed better than the stepwise model from only OCAC data, but included more SNPs.

The observed distribution of the OR estimates within centiles of the PRS distribution were consistent with ORs from predicted values under the assumption that all SNPs interact multiplicatively (Fig. 2), with all 95\% confidence intervals intersecting with the theoretical estimates for women of European ancestries. Compared with women in the middle quintile, women of European ancestry (UK Biobank) in the top 95th percentile of the lasso derived PRS model had a 2.23 -fold increased odds of nonmucinous EOC (95\% Cl: 1.64 - 3.02) (Table 3).

Absolute risk of developing ovarian cancer by PRS percentiles We estimated cumulative risk of EOC within PRS percentiles for women in the general population (Fig. 3), by applying the odds

Table 1. Performance of different PRS models in five-fold cross-validation of OCAC data.

\begin{tabular}{|c|c|c|c|c|c|}
\hline Model & Number of SNPs ${ }^{a}$ & Tuning parameter for best performance & AUC & OR per 1 SD of PRS & $95 \% \mathrm{Cl}$ \\
\hline \multicolumn{6}{|c|}{ (a) Models based on individual level genotype data } \\
\hline Lasso & 1403 & $\lambda=3.3$ & 0.583 & 1.35 & $1.30-1.39$ \\
\hline Elastic net & 10,797 & $\lambda=3.3, k=-2.2$ & 0.586 & 1.36 & $1.31-1.40$ \\
\hline MCP & 1403 & $\lambda=3.3$ & 0.583 & 1.35 & $1.30-1.39$ \\
\hline \multicolumn{6}{|c|}{ (b) Models based on summary statistics } \\
\hline LDPred & $5,291,719$ & $\rho=0.001$ & 0.552 & 1.21 & $1.13-1.29$ \\
\hline
\end{tabular}

$A U C$ area under the receiver operating characteristic (ROC) curve AUC), OR odds ratio, SD standard deviation, $P R S$ polygenic risk score, $C l$ confidence interval, NA not applicable.

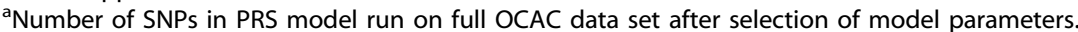

Table 2. External validation of PRS models in European populations using data from UK Biobank and CIMBA.

\begin{tabular}{|c|c|c|c|c|c|c|c|c|c|c|}
\hline \multirow[t]{2}{*}{ Model (data set) } & \multirow[t]{2}{*}{ SNPs } & \multicolumn{3}{|c|}{ UK Biobank } & \multicolumn{3}{|c|}{ CIMBA BRCA1 carriers $^{\mathrm{a}}$} & \multicolumn{3}{|c|}{ 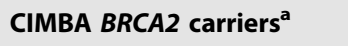 } \\
\hline & & AUC & OR & $95 \% \mathrm{Cl}$ & AUC & HR & $95 \% \mathrm{Cl}$ & AUC & HR & $95 \% \mathrm{Cl}$ \\
\hline \multicolumn{11}{|l|}{ (a) PRS models based on OCAC data } \\
\hline Elastic net (OCAC) & 10,797 & 0.588 & 1.36 & $1.26-1.47$ & 0.583 & 1.32 & $1.26-1.39$ & 0.617 & 1.47 & $1.33-1.63$ \\
\hline Stepwise (OCAC) & 22 & 0.588 & 1.35 & $1.26-1.46$ & 0.563 & 1.21 & $1.16-1.26$ & 0.605 & 1.39 & $1.26-1.54$ \\
\hline Select and shrink (OCAC) & 27,240 & 0.588 & 1.38 & $1.28-1.48$ & 0.592 & 1.36 & $1.29-1.43$ & 0.624 & 1.49 & $1.35-1.64$ \\
\hline Stepwise $(\mathrm{OCAC}-\mathrm{CIMBA})^{\mathrm{b}}$ & 36 & 0.595 & 1.39 & $1.29-1.50$ & NA & NA & NA & NA & NA & NA \\
\hline Select and shrink (OCAC-CIMBA) & 18,007 & 0.596 & 1.42 & $1.32-1.54$ & NA & NA & NA & NA & NA & NA \\
\hline
\end{tabular}

$A U C$ area under the receiver operating characteristic curve, $O R$ odds ratio, $H R$ hazards ratio.

${ }^{\mathrm{a}}$ Estimates are from unadjusted models.

${ }^{\mathrm{b}}$ Results in CIMBA are overfitted as the CIMBA data was used for model development. 

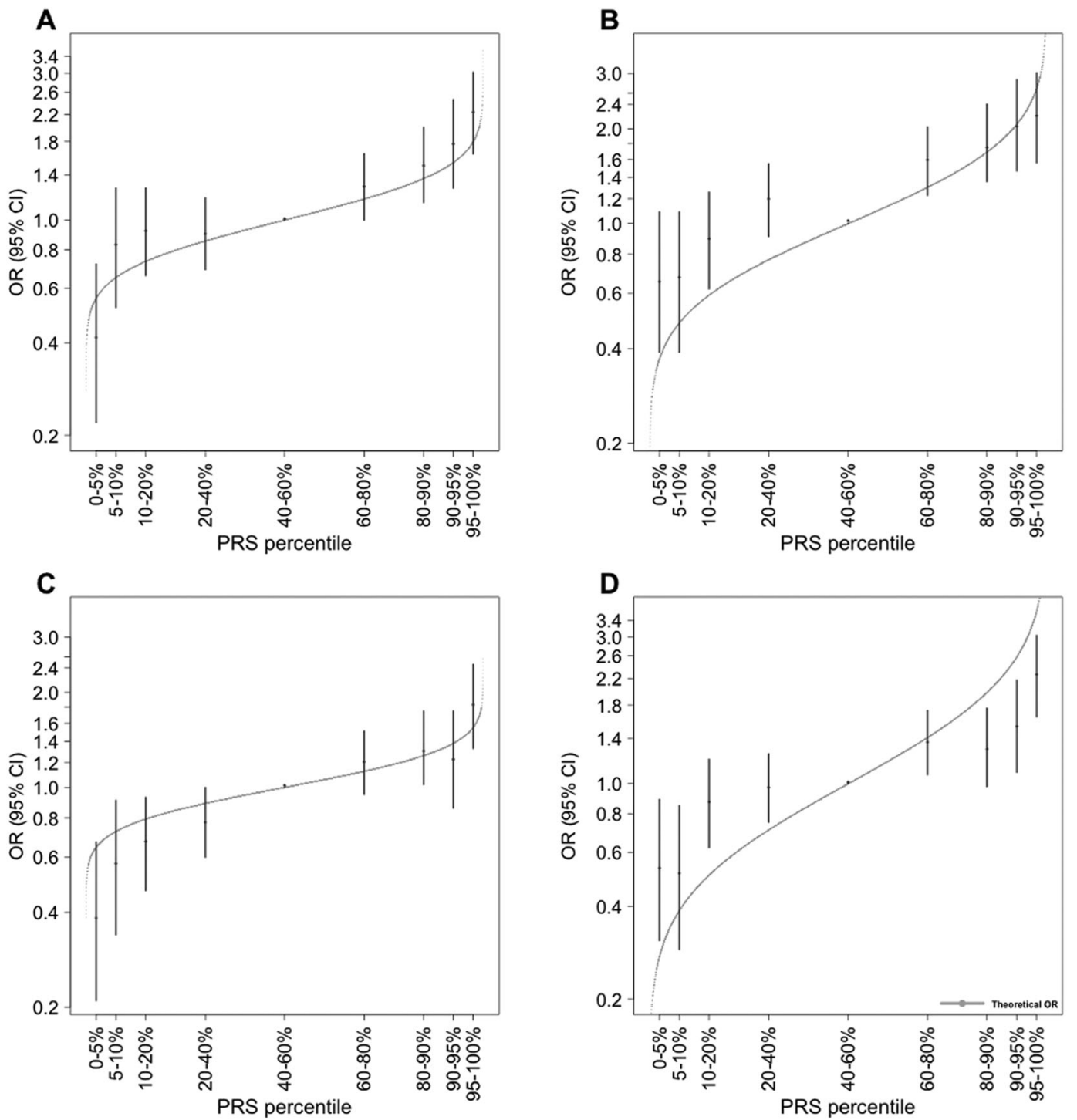

Fig. 2 Association between the PLR PRS models and non-mucinous ovarian cancer by PRS percentiles. Shown are estimated odds ratios $(\mathrm{OR})$ and confidence intervals for women of European ancestries by percentiles of polygenic risk scores derived from lasso (A), elastic net (B), stepwise (C) and S4 (D) models relative to the middle quintile.

ratio from the PRS models to age-specific population incidence and mortality data for England in 2016. For BRCA1 and BRCA2 pathogenic variant carriers, we applied the estimated hazard ratios from PRS models to age-specific incidence rates obtained from Kuchenbaecker et al. [21]. For women in the general population, the estimated cumulative risks of EOC by age 80 for women at the 99th centile of the PRS distribution were $2.24 \%$, $2.18 \%, 2.54 \%$, and $2.81 \%$ for the lasso, elastic net, stepwise and S4 models, respectively. In comparison, the absolute risks of EOC by age 80 for women at the 1 st centile were $0.76 \%, 0.78 \%, 0.64 \%$, and $0.56 \%$ for the lasso, elastic net, stepwise and S4 models, respectively.

The absolute risks of developing EOC in BRCA1 and BRCA2 pathogenic variant carriers were considerably higher than for women in the general population (Figs. S1 and S2). The estimated absolute risk of developing ovarian cancer by age 80 for $B R C A 1$ carriers at the 99th PRS centiles were $63.2 \%, 66.3 \%, 59.0 \%$, and $68.4 \%$ for the lasso, elastic net, stepwise and S4 models, respectively. The corresponding absolute risks for women at the 1st PRS centile were $27.7 \%, 25.6 \%, 30.8 \%$, and $24.2 \%$. For BRCA2 carriers the absolute risks for women at the 99th centile were
$36.3 \%, 36.3 \%, 33.0 \%$, and $36.9 \%$; and $7.10 \%, 7.12 \%, 8.24 \%$, and $6.92 \%$ at the 1 st centile for the lasso, elastic net, stepwise and S4 models, respectively.

\section{PRS distribution and ancestries}

To investigate the transferability of the PRS to other populations, we applied the scores to women of African $(N=1,072)$ and Asian $(N=7,669)$ ancestries genotyped as part of the OncoArray project. In general, the distributions of the raw PRS were dependent on both the statistical methods used in SNP selection and ancestral group. PRS models that included more variants had less dispersion, such that the elastic net models had the least between individual variation in all ancestral groups (standard deviation = 0.15, 0.19, and 0.22 for individuals of Asian, African and European ancestries respectively), while the distributions from the stepwise models were the most dispersed (standard deviation $=0.23,0.27$, and 0.30 for individuals of Asian, African and European ancestries respectively). As expected, given the variation in variant frequencies by population, the distribution of polygenic scores was significantly different across the three ancestral groups, with the least dispersion among women of Asian ancestries and the most 


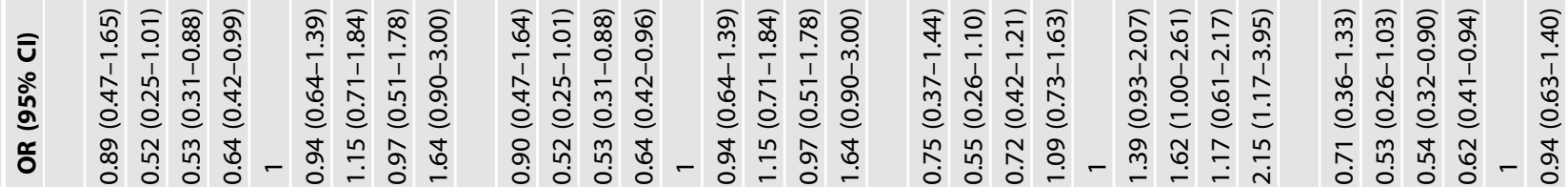

ड

ำ 요

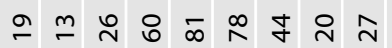

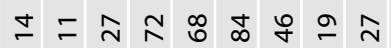

$\stackrel{m}{\sim} \stackrel{\infty}{\infty} \infty \wedge$

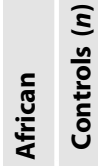

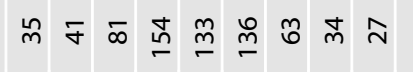

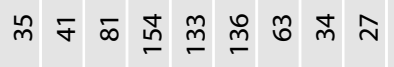

ำ

$\stackrel{m}{m} \bar{\sigma} \stackrel{\circ}{\stackrel{\sim}{m}} \stackrel{\underline{m}}{m}$

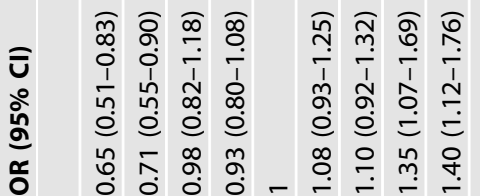

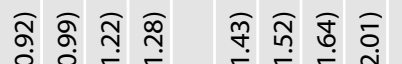

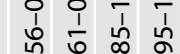

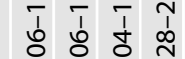

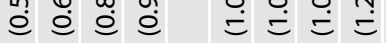

苟

र

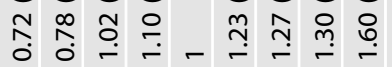

e 0

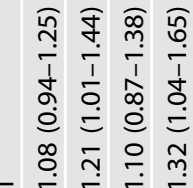

कळ

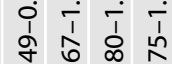

ণิ

负

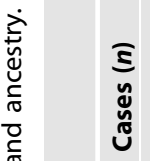

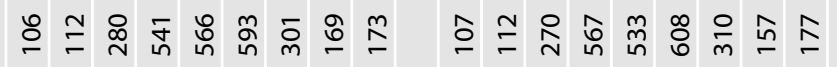

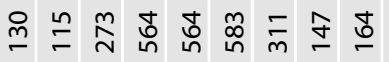

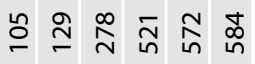

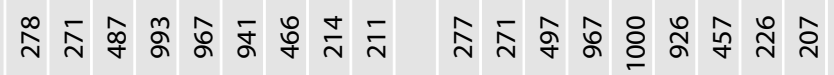

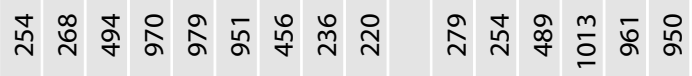

๙ิำ

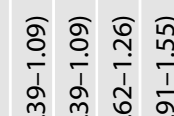

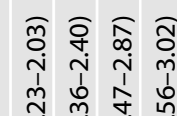

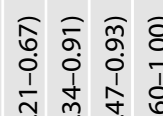

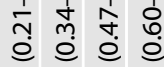

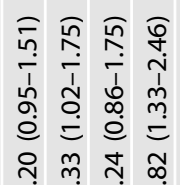

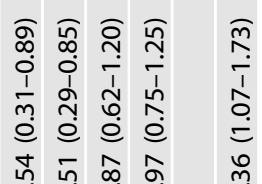

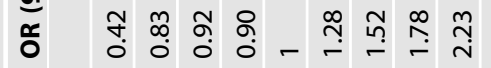

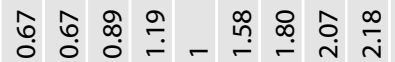

塞

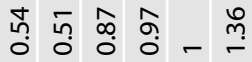

$\Xi$
$y$
$\tilde{~}$

$\simeq$ ป็ก

ニะ ช ํํํำ

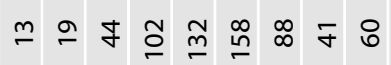

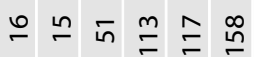

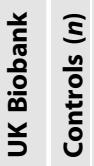

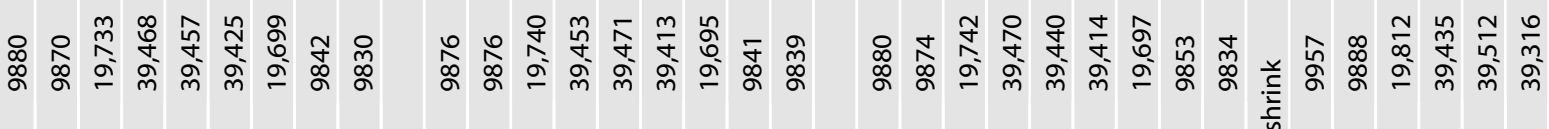
竞 
variation in women of European ancestries. The difference in PRS distribution was minimized after correction for ancestry by standardizing the PRS to have unit standard deviation using the control subjects for each ancestral group.

High PRSs were significantly associated with risk of nonmucinous EOC in both Asian and African ancestries (Table 4), although the effects were weaker than in women of European ancestries. For example, with the lasso model, the odds ratio per unit standard deviation increment in polygenic score was 1.16 (95\% Cl: 1.11-1.22) in women of East Asian ancestries, 1.28 (95\% Cl: $1.13-1.45)$ in women of African ancestries and $1.37(95 \%$ Cl: 1.27-1.48) in women of European ancestries ( $p$ for heterogeneity $<0.0001)$. Variability in effect sizes among ancestral groups was highest for the stepwise model $\left(I^{2}=92 \%\right)$ versus $84 \%$ and $83 \%$ for elastic net and lasso derived polygenic scores respectively. The best discriminative model among women of East Asian and African ancestries were the elastic net PRS (AUC $=0.543$ ) and the S4 PRS derived from OCAC-CIMBA meta-analysis (AUC= $0.596)$ respectively. Women of African ancestries in the top $5 \%$ of the PRS had about two-fold increased risk compared to women in the middle quintile (lasso OR: 1.64, 95\% Cl: 0.90-3.00; elastic net OR: 1.64, 95\% Cl: 0.90-3.00; stepwise OR: 2.15, 95\% Cl: 1.17-3.95; S4 OR: 1.80, 95\% Cl: 0.99-3.31) (Table 3). Effect estimates were smaller in women of East Asian ancestries with women in the top $5 \%$ of the PRS, having about a 1.5 fold increased risk compared to women in the middle quintile (lasso OR: $1.40,95 \% \mathrm{Cl}: 1.12-1.76$; elastic net OR: $1.60,95 \% \mathrm{Cl}: 1.28-2.01$; stepwise OR: $1.32,95 \% \mathrm{Cl}$ : 1.04-1.65; S4 OR: 1.32, 95\% Cl: 1.05-1.66) (Table 3).

\section{DISCUSSION}

Genetic risk profiling with PRSs has led to actionable outcomes for cancers such as breast and prostate [22, 23]. Previous PRS scores for invasive $E O C$ risk in the general population and $B R C A 1 / B R C A 2$ pathogenic variant carriers have been based on genetic variants for which an association with EOC risk had been established at nominal genome-wide significance $[20,24,25]$. Here, we explored the predictive performance of computationally efficient, penalized, regression methods in modeling joint SNP effects for EOC risk prediction in diverse populations and compared them with common approaches. By leveraging the correlation between SNPs which do not reach nominal genome-wide thresholds and including them in PRS models, the PRSs derived from penalized regression models provide stronger evidence of association with risk of nonmucinous EOC than previously published PRSs in both the general population and in $B R C A 1 / B R C A 2$ pathogenic variant carriers.

Recently, Barnes et al. derived a PRS score using 22 SNPs that were significantly associated with high-grade serous EOC risk $\left(\mathrm{PRS}_{\mathrm{HGS}}\right)$ to predict EOC risk in BRCA1/BRCA2 pathogenic variant carriers [20]. To make effect estimates obtained in this analysis comparable to the effect estimates obtained from the $\mathrm{PRS}_{\mathrm{HGS}}$, we standardized all PRSs using the standard deviation from unaffected $B R C A 1 / B R C A 2$ carriers and provide estimates which are directly comparable to the PRS $\mathrm{HGS}_{\mathrm{H}}$ in Supplementary Table 9. All PRS models in this analysis except the Stepwise (OCAC only) had higher effect estimates [20]. The AUC estimates from the adjusted PLR methods implemented in this analysis, are higher than the corresponding $\mathrm{PRS}_{\mathrm{HGS}}$ estimates for BRCA1 carriers (0.604). In BRCA2 carriers, the AUC estimates for the lasso and S4 models did slightly better than the PRS ${ }_{\text {HGS }}$ AUC estimate (0.667), while the stepwise did slightly worse and the elastic net estimate was comparable. The AUC estimates for women in the general population, as estimated from the UK Biobank, are slightly higher than estimates from previously published PRS models for overall EOC risk by Jia et al. (AUC $=0.57$ ) and Yang et al. (AUC $=0.58)[25,26]$.

The level of risk for women above the 95th percentile of the PRS is similar to that conferred by pathogenic variants in moderate penetrance genes such as FANCM $(\mathrm{RR}=2.1,95 \% \mathrm{Cl}=1.1-3.9)$ and 

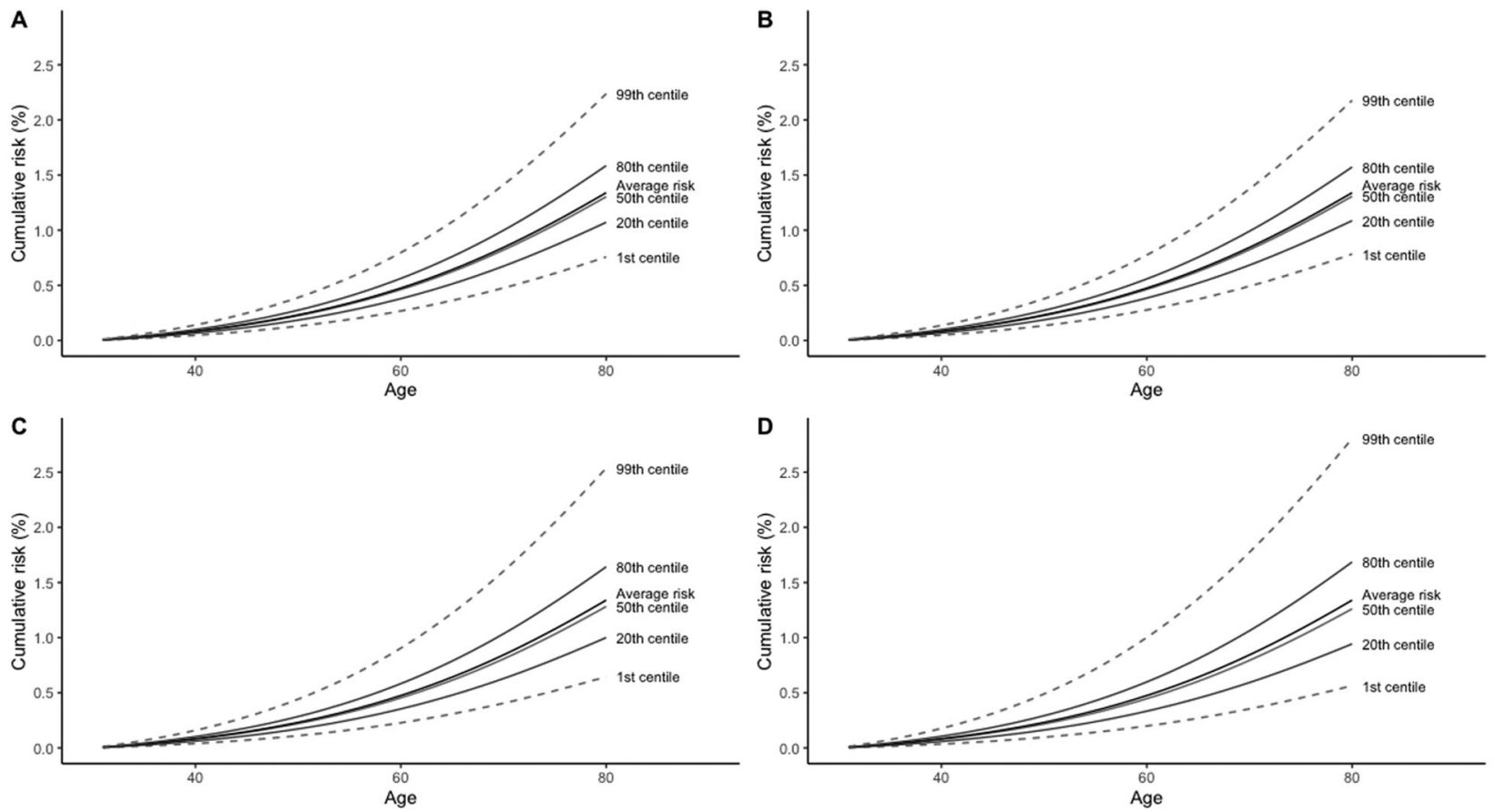

Fig. 3 Cumulative risk of ovarian cancer between birth and age $\mathbf{8 0}$ by PRS percentiles and PRS models. Shown are the cumulative risk of ovarian cancer risk in UK women by polygenic risk score percentiles. The lasso (A) and elastic net (B) penalized regression models were applied to individual level genotype data, while the stepwise (C) and S4 (D) models were applied to summary level statistics. Note that the median and the mean risk differ because the distribution of the relative risk in the population is left-skewed (the log relative risk is a Normal distribution).

Table 4. External validation of PRS models in East Asian and African Populations.

\begin{tabular}{|c|c|c|c|c|c|c|}
\hline \multirow[t]{2}{*}{ Model } & \multicolumn{2}{|c|}{ East Asian ancestries } & \multicolumn{4}{|c|}{ African ancestries } \\
\hline & AUC & OR & $95 \% \mathrm{Cl}$ & AUC & OR & $95 \% \mathrm{Cl}$ \\
\hline Lasso & 0.541 & 1.16 & $(1.11-1.22)$ & 0.576 & 1.28 & $(1.13-1.45)$ \\
\hline Stepwise (OCAC) & 0.528 & 1.11 & $(1.06-1.16)$ & 0.581 & 1.34 & $(1.18-1.52)$ \\
\hline Select and shrink (OCAC) & 0.538 & 1.14 & $(1.08-1.19)$ & 0.593 & 1.38 & $(1.21-1.58)$ \\
\hline Stepwise (OCAC-CIMBA) & 0.542 & 1.17 & $(1.11-1.23)$ & 0.594 & 1.37 & $(1.20-1.56)$ \\
\hline
\end{tabular}

PALB2 (RR $=2.9195 \% \mathrm{Cl}=1.40-6.04)[27,28]$. The inclusion of other risk factors such as family history of ovarian cancer, presence of rare pathogenic variants, age at menarche, oral contraceptive use, hormone replacement therapy, parity, and endometriosis in combination with the PRS could potentially improve risk stratification as implemented in the CanRisk tool (www.canrisk. org), which currently uses a 36-SNP PRS with the potential to use other PRS models [29, 30].

We found that the discrimination of the PRS varied by ancestry with greater discrimination in women of European ancestries than in women of African and East Asian ancestries. The better performance in African than East Asian populations is in contrast to what one would expect given human demographic history, and the performance of PRS for other phenotypes in African populations. This may simply be the play of chance given the small number of samples of African ancestries. Alternatively it reflects the fact that the allele frequencies of the PRS SNPs were more similar between the African and European populations than they were with the East Asian population (Supplementary Tables 10-14).

Further optimization of the models could be achieved by varying the penalization function based on prior knowledge. For example, varying the penalty function to select more SNPs from genomic regions with known susceptibility variants given that susceptibility variants tend to cluster together. Alternatively, the penalty functions could be modified to incorporate information about functionally active regions of the genome such a promoters, enhancers, and transcription factor binding sites. However, incorporating functional annotation has resulted in limited gains in prediction accuracy for complex traits such as breast cancer, celiac disease, type 2 diabetes, and rheumatoid arthritis [31].

Machine/deep learning approaches are alternative ways to constructing PRS, but methods such as the neural net, support vector machine, and random forest have been shown to be computationally prohibitive or produce inferior results to other approaches $[32,33]$. Other machine learning methods, such as those based on gradient boosting do not perform well in genomic regions where strong genetic interactions are present, for which alternative approaches such as the LDPred may perform better [18]. Our approach has several benefits over alternative machine learning methods, including its simplicity, and intrinsic robustness to minor misspecification of LD or association strength. 
In conclusion, our results indicate that using the lasso model for individual level genotype data and the S4 model for summary level data in PRS construction provide an improvement in risk prediction for non-mucinous EOC over more common approaches. Our approach overcomes the computational limitations in the use of penalized methods for large-scale genetic data, particularly in the presence of highly correlated SNPs and when the use of cross-validation for parameter estimation is preferred. In practical terms, the PRS provides sufficient discrimination, particularly for women of European ancestries, to be considered for inclusion in risk prediction and prevention approaches for EOC in the future. Further studies are required to optimize these PRSs in ancestrally diverse populations and to validate their performance with the inclusion of other genetic and lifestyle risk factors.

\section{DATA AVAILABILITY}

OncoArray germline genotype data for the OCAC studies have been deposited the European Genome-phenome Archive (EGA; https://ega-archive.org/), which is hosted by the EBI and the CRG, under accession EGAS00001002305. Summary statisitics for the Ovarian Cancer Association Consortium are available in the NHGRI-EBI GWAS catalogue (https://www.ebi.ac.uk/gwas/home) under the accession number GCST90016665. A subset of the OncoArray germline genotype data for the CIMBA studies are publically available through the database of Genotypes and Phenotypes (dbGaP) under accession phs001321.v1.p1. The complete data set will not be made publically available because of restraints imposed by the ethics committees of individual studies; requests for further data can be made to the Data Access Coordination Committee (http://cimba.ccge.medschl.cam.ac.uk/)

\section{REFERENCES}

1. Jones MR, Kamara D, Karlan BY, Pharoah PDP, Gayther SA. Genetic epidemiology of ovarian cancer and prospects for polygenic risk prediction. Gynecol Oncol. 2017;147:705-13.

2. Lyra PCM, Rangel LB, Monteiro ANA. Functional landscape of common variants associated with susceptibility to epithelial ovarian cancer. Curr Epidemiol Rep. 2020;7:49-57

3. Kar SP, Berchuck A, Gayther SA, Goode EL, Moysich KB, Pearce CL, et al. Common genetic variation and susceptibility to ovarian cancer: current insights and future directions. Cancer Epidemiol Biomark Prev Publ Am Assoc Cancer Res Cosponsored Am Soc Prev Oncol. 2018;27:395-404.

4. Wray NR, Goddard ME, Visscher PM. Prediction of individual genetic risk to disease from genome-wide association studies. Genome Res. 2007;17:1520-8.

5. Purcell SM, Wray NR, Stone JL, Visscher PM, O'Donovan MC, International Schizophrenia Consortium. et al. Common polygenic variation contributes to risk of schizophrenia and bipolar disorder. Nature. 2009;460:748-52.

6. Abraham G, Kowalczyk A, Zobel J, Inouye M. Performance and robustness of penalized and unpenalized methods for genetic prediction of complex human disease. Genet Epidemiol. 2013;37:184-95.

7. Habier D, Fernando RL, Kizilkaya K, Garrick DJ. Extension of the bayesian alphabet for genomic selection. BMC Bioinformatics. 2011;12:186.

8. Szymczak S, Biernacka JM, Cordell HJ, González-Recio O, König IR, Zhang H, et al. Machine learning in genome-wide association studies. Genet Epidemiol. 2009;33: S51-57.

9. Privé $F$, Aschard $H$, Blum MGB. Efficient implementation of penalized regression for genetic risk prediction. Genetics. 2019;212:65-74.

10. Mak TSH, Porsch RM, Choi SW, Zhou X, Sham PC. Polygenic scores via penalized regression on summary statistics. Genet Epidemiol. 2017;41:469-80.

11. Perren TJ. Mucinous epithelial ovarian carcinoma. Ann Oncol Off J Eur Soc Med Oncol. 2016;27:i53-7.

12. Phelan CM, Kuchenbaecker KB, Tyrer JP, Kar SP, Lawrenson K, Winham SJ, et al. Identification of 12 new susceptibility loci for different histotypes of epithelial ovarian cancer. Nat Genet. 2017;49:680-91.

13. Bycroft C, Freeman C, Petkova D, Band G, Elliott LT, Sharp K, et al. The UK Biobank resource with deep phenotyping and genomic data. Nature. 2018;562:203-9.

14. Lawrenson K, Song F, Hazelett DJ, Kar SP, Tyrer J, Phelan CM, et al. Genome-wide association studies identify susceptibility loci for epithelial ovarian cancer in east Asian women. Gynecol Oncol. 2019;153:343-55.

15. Manichaikul A, Peres LC, Wang X-Q, Barnard ME, Chyn D, Sheng X, et al. Identification of novel epithelial ovarian cancer loci in women of African ancestry. Int J Cancer. 2020;146:2987-98.
16. Phelan CM, Kuchenbaecker KB, Tyrer JP, Kar SP, Lawrenson K, Winham SJ, et al. Identification of 12 new susceptibility loci for different histotypes of epithelial ovarian cancer. Nat Genet. 2017;49:680-91.

17. Yang J, Ferreira T, Morris AP, Medland SE, Genetic Investigation of ANthropometric Traits (GIANT) Consortium, DIAbetes Genetics Replication And Metaanalysis (DIAGRAM) Consortium. et al. Conditional and joint multiple-SNP analysis of GWAS summary statistics identifies additional variants influencing complex traits. Nat Genet. 2012;44:369-75. S1-3

18. Vilhjálmsson BJ, Yang J, Finucane HK, Gusev A, Lindström S, Ripke S, et al. Modeling linkage disequilibrium increases accuracy of polygenic risk scores. Am J Hum Genet. 2015;97:576-92.

19. Ge T, Chen C-Y, Ni Y, Feng Y-CA, Smoller JW. Polygenic prediction via Bayesian regression and continuous shrinkage priors. Nat Commun. 2019;10:1776.

20. Barnes DR, Rookus MA, McGuffog L, Leslie G, Mooij TM, Dennis J, et al. Polygenic risk scores and breast and epithelial ovarian cancer risks for carriers of BRCA1 and BRCA2 pathogenic variants. Genet Med Off J Am Coll Med Genet. 2020;15:576-92.

21. Kuchenbaecker KB, Hopper JL, Barnes DR, Phillips K-A, Mooij TM, Roos-Blom M-J, et al. Risks of Breast, Ovarian, and Contralateral Breast Cancer for BRCA1 and BRCA2 Mutation Carriers. JAMA. 2017;317:2402-16.

22. Mavaddat N, Michailidou K, Dennis J, Lush M, Fachal L, Lee A, et al. Polygenic risk scores for prediction of breast cancer and breast cancer subtypes. Am J Hum Genet. 2019;104:21-34.

23. Schumacher FR, Al Olama AA, Berndt SI, Benlloch S, Ahmed M, Saunders EJ, et al. Association analyses of more than 140,000 men identify 63 new prostate cancer susceptibility loci. Nat Genet. 2018;50:928-36.

24. Kuchenbaecker KB, McGuffog L, Barrowdale D, Lee A, Soucy P, Dennis J, et al. Evaluation of Polygenic Risk Scores for Breast and Ovarian Cancer Risk Prediction in BRCA1 and BRCA2 Mutation Carriers. J Natl Cancer Inst. 2017;109.

25. Yang X, Leslie G, Gentry-Maharaj A, Ryan A, Intermaggio M, Lee A, et al. Evaluation of polygenic risk scores for ovarian cancer risk prediction in a prospective cohort study. J Med Genet. 2018;55:546-54.

26. Jia G, Lu Y, Wen W, Long J, Liu Y, Tao R, et al. Evaluating the utility of polygenic risk scores in identifying high-risk individuals for eight common cancers. JNCI Cancer Spectr. 2020;4:pkaa021.

27. Song $H$, Dicks EM, Tyrer J, Intermaggio $M$, Chenevix-Trench $G$, Bowtell DD, et al. Population-based targeted sequencing of 54 candidate genes identifies PALB2 as a susceptibility gene for high-grade serous ovarian cancer. J Med Genet. 2021;58:305-13.

28. Yang X, Leslie G, Doroszuk A, Schneider S, Allen J, Decker B, et al. Cancer risks associated with germline PALB2 pathogenic variants: an international study of 524 families. J Clin Oncol Off J Am Soc Clin Oncol. 2020;38:674-85.

29. Lee A, Mavaddat N, Wilcox AN, Cunningham AP, Carver T, Hartley S, et al. BOADICEA: a comprehensive breast cancer risk prediction model incorporating genetic and nongenetic risk factors. Genet Med Off J Am Coll Med Genet. 2019;21:1708-18.

30. Welcome to CanRisk. [cited 2021 Aug 15]. https://canrisk.org/

31. Hu Y, Lu Q, Powles R, Yao X, Yang C, Fang F, et al. Leveraging functional annotations in genetic risk prediction for human complex diseases. PLoS Comput Biol. 2017;13:e1005589.

32. Gola D, Erdmann J, Müller-Myhsok B, Schunkert H, König IR. Polygenic risk scores outperform machine learning methods in predicting coronary artery disease status. Genet Epidemiol. 2020;44:125-38.

33. Paré G, Mao S, Deng WQ. A machine-learning heuristic to improve gene score prediction of polygenic traits. Sci Rep. 2017;7:12665.

\section{ACKNOWLEDGEMENTS}

See Supplementary Material.

\section{AUTHOR CONTRIBUTIONS}

EO Dareng, JP Tyrer, DR Barnes, MR Jones, X Yang, KK Aben, MA Adank, S Agata, IL Andrulis, H Anton-Culver, NN Antonenkova, G Aravantinos, BK Arun, A Augustinsson, J Balmaña, RB Barkardottir, D Barrowdale, MW Beckmann, A Beeghly-Fadiel, J Benitez, M Bermisheva, MQ Bernardini, L Bjorge, NV Bogdanova, B Bonanni, A Borg, JD Brenton, A Budzilowska, R Butzow, SS Buys, H Cai, MA Caligo, I Campbell, R Cannioto, H Cassingham, J Chang-Claude, SJ Chanock, K Chen, Y Chiew, WK Chung, KB Claes, S Colonna, LS Cook, FJ Couch, MB Daly, F Dao, E Davies, M de la Hoya, R de Putter, J Dennis, A DePersia, P Devilee, O Diez, Y Ding, JA Doherty, SM Domchek, T Dörk, A du Bois, M Dürst, DM Eccles, HA Eliassen, C Engel, D Evans, PA Fasching, JM Flanagan, RT Fortner, E Machackova, E Friedman, PA Ganz, J Garber, F Gensini, GG Giles, G Glendon, AK Godwin, MT Goodman, MH Greene, J Gronwald, E Hahnen, CA Haiman, N Håkansson, U Hamann, TV Hansen, HR Harris, M Hartman, F Heitz, MA Hildebrandt, 
E Høgdall, CK Høgdall, JL Hopper, R Huang, C Huff, PJ Hulick, DG Huntsman, EN Imyanitov, C Isaacs, A Jakubowska, PA James, R Janavicius, A Jensen, OT Johannsson, EM John, ME Jones, D Kang, BY Karlan, A Karnezis, LE Kelemen, E Khusnutdinova, LA Kiemeney, B Kim, SK Kjaer, I Komenaka, J Kupryjanczyk, AW Kurian, A Kwong, D Lambrechts, MC Larson, C Lazaro, ND Le, G Leslie, J Lester, F Lesueur, DA Levine, J Li, JT Loud, KH Lu, J Lubi ski, PL Mai, S Manoukian, JR Marks, R Matsuno, K Matsuo, T May, L McGuffog, JR McLaughlin, IA McNeish, N Mebirouk, A Miller, RL Milne, A Minlikeeva, F Modugno, M Montagna, KB Moysich, E Munro, KL Nathanson, SL Neuhausen, H Nevanlinna, J Ngeow Yuen Yie, H Nielsen, L Nikitina-Zake, K Odunsi, K Offit, E Olah, S Olbrecht, OI Olopade, SH Olson, H Olsson, A Osorio, L Papi, SK Park, MT Parsons, H Pathak, I Pedersen, A Peixoto, T Pejovic, P Perez-Segura, JB Permuth, B Peshkin, P Peterlongo, A Piskorz, D Prokofyeva, P Radice, J Rantala, MJ Riggan, HA Risch, C Rodriguez-Antona, E Ross, M Rossing, I Runnebaum, DP Sandler, M Santamariña, P Soucy, RK Schmutzler, V Setiawan, K Shan, W Sieh, J Simard, CF Singer, AP Sokolenko, H Song, MC Southey, H Steed, D Stoppa-Lyonnet, R Sutphen, AJ Swerdlow, Y Tan, MR Teixeira, S Teo, KL Terry, M Terry, M Thomassen, PJ Thompson, L Thomsen, DL Thull, M Tischkowitz, L Titus, AE Toland, D Torres, B Trabert, R Travis, N Tung, SS Tworoger, E Valen, AM van Altena, AH van der Hout, E Van Nieuwenhuysen, EJ van Rensburg, A Vega, D Velez Edwards, RA Vierkant, F Wang, PM Webb, CR Weinberg, JN Weitzel, N Wentzensen, E White, SJ Winham, A Wolk, Y Woo, AH Wu, L Yan, D Yannoukakos, KM Zavaglia, W Zheng, A Ziogas, KK Zorn, K Lawrenson, TA Sellers, SJ Ramus, AN Monteiro, JM Cunningham, EL Goode, JM Schildkraut, A Berchuck, G Chenevix-Trench, SA Gayther, AC Antoniou, PD Pharoah contributed and/or designed the work that led to this submission, acquired data, played important roles in interpreting results, drafted or revised the manuscript, approved the final version and agreed to be accountable for all aspects of the work.

\section{COMPETING INTERESTS}

ADF has received a research grant from AstraZeneca, not directly related to the content of this manuscript. MWB conducts research funded by Amgen, Novartis and Pfizer. PAF conducts research funded by Amgen, Novartis and Pfizer. He received Honoraria from Roche, Novartis and Pfizer. AWK reports research funding to her institution from Myriad Genetics for an unrelated project. UM owns stocks in Abcodia Ltd. Rachel A. Murphy is a consultant for Pharmavite. The other authors declare no conflicts of interest.

\section{ETHICS STATEMENT}

All study participants provided written informed consent and participated in research or clinical studies at the host institute under ethically approved protocols. The studies and their approving institutes are listed in the Supplementary Material (Ethics Statement).

\section{ADDITIONAL INFORMATION}

Supplementary information The online version contains supplementary material available at https://doi.org/10.1038/s41431-021-00987-7.

Correspondence and requests for materials should be addressed to Paul D. P. Pharoah.

Reprints and permission information is available at http://www.nature.com/ reprints

Publisher's note Springer Nature remains neutral with regard to jurisdictional claims in published maps and institutional affiliations.

Eileen O. Dareng $\mathbb{D}^{1,243}$, Jonathan P. Tyrer $\mathbb{D}^{2,243}$, Daniel R. Barnes $\mathbb{D}^{1}{ }^{1}$, Michelle R. Jones ${ }^{3}$, Xin Yang $\mathbb{D i D}^{1}$, Katja K. H. Aben ${ }^{4,5}$, Muriel A. Adank $^{6}$, Simona Agata ${ }^{7}$, Irene L. Andrulis (D) $^{8,9}$, Hoda Anton-Culver ${ }^{10}$, Natalia N. Antonenkova ${ }^{11}$, Gerasimos Aravantinos ${ }^{12}$, Banu K. Arun $^{13}$, Annelie Augustinsson (iD) ${ }^{14}$, Judith Balmaña ${ }^{15,16}$, Elisa V. Bandera ${ }^{17}$, Rosa B. Barkardottir ${ }^{18,19}$, Daniel Barrowdale (iD), Matthias W. Beckmann $^{20}$, Alicia Beeghly-Fadiel ${ }^{21}$, Javier Benitez ${ }^{22,23}$, Marina Bermisheva ${ }^{24}$, Marcus Q. Bernardini ${ }^{25}$, Line Bjorge ${ }^{26,27}$, Amanda Black ${ }^{28}$, Natalia V. Bogdanova ${ }^{11,29,30}$, Bernardo Bonanni iD $^{31}$, Ake Borg ${ }^{32}$, James D. Brenton (iD ${ }^{33}$, Agnieszka Budzilowska ${ }^{34}$, Ralf Butzow (iD ${ }^{35}$, Saundra S. Buys ${ }^{36}$, Hui Cai ${ }^{21}$, Maria A. Caligo ${ }^{37}$, lan Campbell (iD ${ }^{38,39}$, Rikki Cannioto ${ }^{40}$, Hayley Cassingham ${ }^{41}$, Jenny Chang-Claude ${ }^{42,43}$, Stephen J. Chanock iD $^{44}$, Kexin Chen ${ }^{45}$, Yoke-Eng Chiew ${ }^{46,47}$, Wendy K. Chung ${ }^{48}$, Kathleen B. M. Claes (iD) ${ }^{49}$, Sarah Colonna ${ }^{36}$, GEMO Study Collaborators $^{50,51,52}$, GC-HBOC Study Collaborators ${ }^{53}$, EMBRACE Collaborators ${ }^{1}$, Linda S. Cook ${ }^{54,55}$, Fergus J. Couch ${ }^{56}$, Mary B. Daly ${ }^{57}$, Fanny Dao ${ }^{58}$, Eleanor Davies ${ }^{59}$, Miguel de la Hoya ${ }^{60}{ }^{60}$, Robin de Putter ${ }^{49}{ }^{49}$, Joe Dennis $\mathbb{I D}^{1}$, Allison DePersia ${ }^{61,62}$, Peter Devilee ID $^{63,64}$, Orland Diez ${ }^{65,66}$, Yuan Chun Ding ${ }^{67}$, Jennifer A. Doherty ${ }^{68}$, Susan M. Domchek ${ }^{69}$, Thilo Dörk ${ }^{30}$, Andreas du Bois (iD ${ }^{70,71}$, Matthias Dürst ${ }^{72}$, Diana M. Eccles ${ }^{73}$, Heather A. Eliassen ${ }^{74,75}$, Christoph Engel ${ }^{76,77}$, Gareth D. Evans ${ }^{78,79}$, Peter A. Fasching ${ }^{20,80}$, James M. Flanagan ${ }^{81}$, Renée T. Fortner ${ }^{42}$, Eva Machackova (D) $^{82}$, Eitan Friedman ${ }^{83,84}$, Patricia A. Ganz ${ }^{85}$, Judy Garber ${ }^{86}$, Francesca Gensini ${ }^{87}$, Graham G. Giles ${ }^{88,89,90}$, Gord Glendon $^{8}$, Andrew K. Godwin $\mathbb{D}^{91}$, Marc T. Goodman ${ }^{92}$, Mark H. Greene iD $^{93}$, Jacek Gronwald ${ }^{94}$, OPAL Study Group ${ }^{95}$, AOCS Group $^{38,46}$, Eric Hahnen ${ }^{53,96}$, Christopher A. Haiman ${ }^{97}$, Niclas Håkansson ${ }^{98}$, Ute Hamann ${ }^{99}$, Thomas V. O. Hansen ${ }^{100}$, Holly R. Harris ${ }^{101,102}$, Mikael Hartman ${ }^{103,104}$, Florian Heitz (D) $^{70,71,105}$, Michelle A. T. Hildebrandt ${ }^{106}$, Estrid Høgdall ${ }^{107,108}$, Claus K. Høgdall ${ }^{109}$, John L. Hopper ${ }^{89}$, Ruea-Yea Huang ${ }^{110}$, Chad Huff ${ }^{106}$, Peter J. Hulick (DD $^{61,62}$, David G. Huntsman ${ }^{111,112,113,114}$, Evgeny N. Imyanitov ${ }^{115}$, KConFab Investigators $^{38}$, HEBON Investigators ${ }^{116}$, Claudine Isaacs ${ }^{117}$, Anna Jakubowska iD $^{94,118}$, Paul A. James (iD) ${ }^{39,119}$, Ramunas Janavicius ${ }^{120,121}$, Allan Jensen ${ }^{107}$, Oskar Th. Johannsson ${ }^{122}$, Esther M. John ${ }^{123,124}$, Michael E. Jones ${ }^{125}$, Daehee Kang ${ }^{126,127,128}$, Beth Y. Karlan ${ }^{129}$, Anthony Karnezis $^{130}$, Linda E. Kelemen ${ }^{131}$, Elza Khusnutdinova ${ }^{24,132}$, Lambertus A. Kiemeney ID $^{4}$, Byoung-Gie Kim ${ }^{133}$, Susanne K. Kjaer ${ }^{107,109}$, lan Komenaka $^{134}$, Jolanta Kupryjanczyk ${ }^{34}$, Allison W. Kurian (iD ${ }^{123,124}$, Ava Kwong ${ }^{135,136,137}$, Diether Lambrechts (iD ${ }^{138,139}$, Melissa C. Larson ${ }^{140}$, Conxi Lazaro (iD ${ }^{141}$, Nhu D. Le ${ }^{142}$, Goska Leslie (iD) ${ }^{1}$, Jenny Lester ${ }^{129}$, Fabienne Lesueur ${ }^{51,52,143}$, Douglas A. Levine ${ }^{58,144}$, Lian Li ${ }^{45}$, Jingmei $\mathrm{Li}^{145}$, Jennifer T. Loud ${ }^{93}$, Karen H. Lu ${ }^{146}$, Jan Lubiński ${ }^{94}$, Phuong L. Mai ${ }^{10}{ }^{147}$, Siranoush Manoukian ${ }^{148}$, Jeffrey R. Marks ${ }^{149}$, Rayna Kim Matsuno ${ }^{150}$, Keitaro Matsuo (iD ${ }^{151,152}$, Taymaa May ${ }^{25}$, Lesley McGuffog ${ }^{1}$, John R. McLaughlin ${ }^{153}$, lain A. McNeish ${ }^{154,155}$, Noura Mebirouk $^{51,52,143}$, Usha Menon (iD ${ }^{156}$, Austin Miller ${ }^{157}$, Roger L. Milne ${ }^{88,89,90}$, Albina Minlikeeva ${ }^{158}$, Francesmary Modugno ${ }^{159,160}$, Marco Montagna $^{7}$, Kirsten B. Moysich ${ }^{158}$, Elizabeth Munro ${ }^{161,162}$, Katherine L. Nathanson $\mathbb{D}^{69}$, Susan L. Neuhausen ${ }^{67}$, Heli Nevanlinna (D) $^{163}$, 
Joanne Ngeow Yuen Yie ${ }^{10}{ }^{164,165}$, Henriette Roed Nielsen ${ }^{166}$, Finn C. Nielsen ${ }^{100}$, Liene Nikitina-Zake ${ }^{167}$, Kunle Odunsi ${ }^{168}$, Kenneth Offit $^{169,170}$, Edith Olah ${ }^{171}$, Siel Olbrecht ${ }^{172}$, Olufunmilayo I. Olopade ${ }^{173}{ }^{173}$, Sara H. Olson ${ }^{174}$, Håkan Olsson ${ }^{14}$, Ana Osorio ${ }^{23,175}$, Laura Papi $^{87}$, Sue K. Park (D) ${ }^{126,127,128}$, Michael T. Parsons (D) $^{176}$, Harsha Pathak ${ }^{91}$, Inge Sokilde Pedersen (D) ${ }^{177,178,179}$, Ana Peixoto ${ }^{180}$, Tanja Pejovic $^{161,162}$, Pedro Perez-Segura ${ }^{60}$, Jennifer B. Permuth ${ }^{181}$, Beth Peshkin (D) ${ }^{117}$, Paolo Peterlongo (D) ${ }^{182}$, Anna Piskorz ${ }^{33}$, Darya Prokofyeva ${ }^{183}$, Paolo Radice (iD ${ }^{184}$, Johanna Rantala ${ }^{185}$, Marjorie J. Riggan ${ }^{186}$, Harvey A. Risch ${ }^{187}$, Cristina Rodriguez-Antona ${ }^{22,23}$, Eric Ross (D) ${ }^{188}$, Mary Anne Rossing ${ }^{101,102}$, Ingo Runnebaum ${ }^{72}$, Dale P. Sandler (D) ${ }^{189}$, Marta Santamariña ${ }^{175,190,191}$, Penny Soucy ${ }^{192}$, Rita K. Schmutzler ${ }^{53,96,193}$, V. Wendy Setiawan ${ }^{97}$, Kang Shan ${ }^{194}$, Weiva Sieh $\mathbb{D}^{195,196}$, Jacques Simard ${ }^{107}{ }^{197}$, Christian F. Singer ${ }^{198}$, Anna P. Sokolenko ${ }^{115}$, Honglin Song ${ }^{199}$, Melissa C. Southey ${ }^{88,90,200}$, Helen Steed ${ }^{201}$, 'Dominique Stoppa-Lyonnet (iD ${ }^{50,202,203, \text { Rebecca Sutphen }}{ }^{204}$, Anthony J. Swerdlow ${ }^{125,205}$, Yen Yen Tan ${ }^{198}$, Manuel R. Teixeira $\mathbb{D}^{180,206}$, Soo Hwang Teo $\mathbb{1}^{207,208}$, Kathryn L. Terry ${ }^{74,209}$, Mary Beth Terry $^{210}$, The OCAC Consortium ${ }^{186}$, The CIMBA Consortium ${ }^{1}$, Mads Thomassen ${ }^{166}$, Pamela J. Thompson ${ }^{92}$, Liv Cecilie Vestrheim Thomsen ${ }^{26,27}$, Darcy L. Thull ${ }^{211}$, Marc Tischkowitz (iD ${ }^{212,213}$, Linda Titus ${ }^{214}$, Amanda E. Toland (iD ${ }^{215}$, Diana Torres ${ }^{99,216}$, Britton Trabert (D) $^{28,}$ Ruth Travis (D) $^{217}$, Nadine Tung ${ }^{218}$, Shelley S. Tworoger ${ }^{74,181}$, Ellen Valen ${ }^{26,27}$, Anne M. van Altena ${ }^{4}$, Annemieke H. van der Hout ${ }^{219}$, Els Van Nieuwenhuysen ${ }^{172}$, Elizabeth J. van Rensburg $\mathbb{I D}^{220}$, Ana Vega ${ }^{175,221,222}$, Digna Velez Edwards ${ }^{223}$, Robert A. Vierkant ${ }^{140}$, Frances Wang $^{224,225}$, Barbara Wappenschmidt ${ }^{53,96}$, Penelope M. Webb ${ }^{95}{ }^{95}$, Clarice R. Weinberg ${ }^{226}$, Jeffrey N. Weitzel ${ }^{227}$, Nicolas Wentzensen ${ }^{28}$, Emily White ${ }^{102,228}$, Alice S. Whittemore ${ }^{123,229}$, Stacey J. Winham (D) ${ }^{140}$, Alicja Wolk ${ }^{98,230}$, Yin-Ling Woo DD $^{231}$, Anna H. Wu $^{97}$, Li Yan ${ }^{232}$, Drakoulis Yannoukakos (D) ${ }^{233}$, Katia M. Zavaglia ${ }^{37}$, Wei Zheng ${ }^{21}$, Argyrios Ziogas (iD) ${ }^{10}$, Kristin K. Zorn ${ }^{147}$, Zdenek Kleibl (iD ${ }^{234}$, Douglas Easton (iD ${ }^{1,2}$, Kate Lawrenson ${ }^{3,235}$, Anna DeFazio (iD ${ }^{46,47}$, Thomas A. Sellers ${ }^{236}$, Susan J. Ramus (iD ${ }^{237,238}$, Celeste L. Pearce ${ }^{239,240}$, Alvaro N. Monteiro $\mathbb{B}^{181}$, Julie Cunningham $\mathbb{i D}^{241}$, Ellen L. Goode ${ }^{241}$, Joellen M. Schildkraut ${ }^{242}$, Andrew Berchuck ${ }^{186}$, Georgia Chenevix-Trench ${ }^{176}$, Simon A. Gayther $\mathbb{I}^{3}$, Antonis C. Antoniou ${ }^{1}$ and Paul D. P. Pharoah $\mathbb{D}^{1,2 凶}$

${ }^{1}$ University of Cambridge, Centre for Cancer Genetic Epidemiology, Department of Public Health and Primary Care, Cambridge, UK. ${ }^{2}$ University of Cambridge, Centre for Cancer Genetic Epidemiology, Department of Oncology, Cambridge, UK. ${ }^{3}$ Center for Bioinformatics and Functional Genomics, Cedars-Sinai Medical Center, Los Angeles, CA, USA. ${ }^{4}$ Radboud University Medical Center, Radboud Institute for Health Sciences, Nijmegen, The Netherlands. ${ }^{5}$ Netherlands Comprehensive Cancer Organisation, Utrecht, The Netherlands. ${ }^{6}$ The Netherlands Cancer Institute-Antoni van Leeuwenhoek hospital, Family Cancer Clinic, Amsterdam, The Netherlands. ${ }^{7}$ Veneto Institute of Oncology IOV_IRCCS, Immunology and Molecular Oncology Unit, Padua, Italy. ${ }^{8}$ Lunenfeld-Tanenbaum Research Institute of Mount Sinai Hospital, Fred A. Litwin Center for Cancer Genetics, Toronto, ON, Canada. ${ }^{9}$ University of Toronto, Department of Molecular Genetics, Toronto, ON, Canada. ${ }^{10}$ University of California Irvine, Department of Epidemiology, Genetic Epidemiology Research Institute, Irvine, CA, USA. ${ }^{11}$ N.N. Alexandrov Research Institute of Oncology and Medical Radiology, Minsk, Belarus. ${ }^{12 ' A g i i ~ A n a r g i r i ' ~ C a n c e r ~ H o s p i t a l, ~}$ Athens, Greece. ${ }^{13}$ University of Texas MD Anderson Cancer Center, Department of Breast Medical Oncology, Houston, TX, USA. ${ }^{14}$ Lund University, Department of Cancer Epidemiology, Clinical Sciences, Lund, Sweden. ${ }^{15}$ Vall d'Hebron Institute of Oncology, Hereditary cancer Genetics Group, Barcelona, Spain. ${ }^{16}$ University Hospital of Vall d'Hebron, Department of Medical Oncology, Barcelona, Spain. ${ }^{17}$ Rutgers Cancer Institute of New Jersey, Cancer Prevention and Control Program, New Brunswick, NJ, USA. ${ }^{18}$ Landspitali University Hospital, Department of Pathology, Reykjavik, Iceland. ${ }^{19}$ University of Iceland, BMC (Biomedical Centre), Faculty of Medicine, Reykjavik, Iceland. ${ }^{20}$ University Hospital Erlangen, Friedrich-Alexander-University Erlangen-Nuremberg, Department of Gynecology and Obstetrics, Comprehensive Cancer Center ER-EMN, Erlangen, Germany. ${ }^{21}$ Vanderbilt University School of Medicine, Division of Epidemiology, Department of Medicine, Vanderbilt Epidemiology Center, Vanderbilt-Ingram Cancer Center, Nashville, TN, USA. ${ }^{22}$ Biomedical Network on Rare Diseases (CIBERER), Madrid, Spain. ${ }^{23}$ Spanish National Cancer Research Centre (CNIO), Human Cancer Genetics Programme, Madrid, Spain. ${ }^{24}$ Ufa Federal Research Centre of the Russian Academy of Sciences, Institute of Biochemistry and Genetics, Ufa, Russia. ${ }^{25}$ Princess Margaret Hospital, Division of Gynecologic Oncology, University Health Network, Toronto, ON, Canada. ${ }^{26}$ Haukeland University Hospital, Department of Obstetrics and Gynecology, Bergen, Norway. ${ }^{27}$ University of Bergen, Centre for Cancer Biomarkers CCBIO, Department of Clinical Science, Bergen, Norway. ${ }^{28}$ National Cancer Institute, Division of Cancer Epidemiology and Genetics, Bethesda, MD, USA. ${ }^{29}$ Hannover Medical School, Department of Radiation Oncology, Hannover, Germany. ${ }^{30}$ Hannover Medical School, Gynaecology Research Unit, Hannover, Germany. ${ }^{31}$ IEO, European Institute of Oncology IRCCS, Division of Cancer Prevention and Genetics, Milan, Italy. ${ }^{32}$ Lund University and Skåne University Hospital, Department of Oncology, Lund, Sweden. ${ }^{33}$ Cancer Research UK Cambridge Institute, University of Cambridge, Cambridge, UK. ${ }^{34}$ Maria Sklodowska-Curie National Research Institute of Oncology, Department of Pathology and Laboratory Diagnostics, Warsaw, Poland. ${ }^{35}$ University of Helsinki, Department of Pathology, Helsinki University Hospital, Helsinki, Finland. ${ }^{36} \mathrm{Huntsman}$ Cancer Institute, Department of Medicine, Salt Lake City, UT, USA. ${ }^{37}$ University Hospital, SOD Genetica Molecolare, Pisa, Italy. ${ }^{38}$ Peter MacCallum Cancer Center, Melbourne, VIC, Australia. ${ }^{39}$ The University of Melbourne, Sir Peter MacCallum Department of Oncology, Melbourne, VIC, Australia. ${ }^{40}$ Roswell Park Cancer Institute, Cancer Pathology \& Prevention, Division of Cancer Prevention and Population Sciences, Buffalo, NY, USA. ${ }^{41}$ Division of Human Genetics, The Ohio State University, Department of Internal Medicine, Columbus, OH, USA. ${ }^{42}$ German Cancer Research Center (DKFZ), Division of Cancer Epidemiology, Heidelberg, Germany. ${ }^{43}$ University Medical Center Hamburg-Eppendorf, Cancer Epidemiology Group, University Cancer Center Hamburg (UCCH), Hamburg, Germany. ${ }^{44}$ National Cancer Institute, National Institutes of Health, Department of Health and Human Services, Division of Cancer Epidemiology and Genetics, Bethesda, MD, USA. ${ }^{45}$ Tianjin Medical University Cancer Institute and Hospital, Department of Epidemiology, Tianjin, China. ${ }^{46}$ The University of Sydney, Centre for Cancer Research, The Westmead Institute for Medical Research, Sydney, NSW, Australia. ${ }^{47}$ Westmead Hospital, Department of Gynaecological Oncology, Sydney, NSW, Australia. ${ }^{48}$ Columbia University, Departments of Pediatrics and Medicine, New York, NY, USA. ${ }^{49}$ Ghent University, Centre for Medical Genetics, Gent, Belgium. ${ }^{50}$ INSERM U830, Department of Tumour Biology, Paris, France. ${ }^{51}$ Institut Curie, Paris, France. ${ }^{52}$ Mines ParisTech, Fontainebleau, France. ${ }^{53}$ Faculty of Medicine and University Hospital Cologne, University of Cologne, Center for Familial Breast and Ovarian Cancer, Cologne, Germany. ${ }^{54}$ University of New Mexico, University of New Mexico Health Sciences Center, Albuquerque, NM, USA. ${ }^{55}$ Alberta Health Services, Department of Cancer Epidemiology and Prevention Research, Calgary, AB, Canada. ${ }^{56}$ Mayo Clinic, Department of Laboratory Medicine and Pathology, Rochester, MN, USA. ${ }^{57}$ Fox Chase Cancer Center, Department of Clinical Genetics, Philadelphia, PA, USA. ${ }^{58}$ Memorial Sloan Kettering Cancer Center, Gynecology Service, Department of Surgery, New York, NY, USA. ${ }^{59}$ Cambridge, Cambridge, UK. ${ }^{60}$ CIBERONC, Hospital Clinico San Carlos, IdISSC (Instituto de Investigación Sanitaria del Hospital Clínico San Carlos), Molecular Oncology Laboratory, Madrid, Spain. ${ }^{61}$ NorthShore University Health System, Center for Medical Genetics, Evanston, IL, USA. ${ }^{62}$ The University of Chicago Pritzker School of Medicine, Chicago, IL, USA. ${ }^{63}$ Leiden University Medical Center, Department of Pathology, Leiden, The Netherlands. ${ }^{64}$ Leiden University Medical Center, Department of Human Genetics, Leiden, The Netherlands. ${ }^{65}$ Vall dHebron Institute of Oncology (VHIO), Oncogenetics Group, Barcelona, Spain. ${ }^{66}$ University Hospital Vall dHebron, Clinical and Molecular Genetics Area, Barcelona, Spain. ${ }^{67}$ Beckman Research Institute of City of Hope, Department of Population Sciences, Duarte, CA, USA. ${ }^{68}$ University of Utah, Huntsman Cancer Institute, Department of Population Health Sciences, Salt Lake City, UT, USA. ${ }^{69}$ University of Pennsylvania, Basser Center for BRCA, Abramson Cancer Center, Philadelphia, PA, USA. ${ }^{70}$ Ev. Kliniken Essen-Mitte (KEM), Department of Gynecology and Gynecologic Oncology, Essen, Germany. ${ }^{71}$ Dr. Horst Schmidt Kliniken Wiesbaden, Department of Gynecology and Gynecologic Oncology, Wiesbaden, Germany. ${ }^{72}$ Jena University Hospital一Friedrich Schiller University, Department of Gynaecology, Jena, Germany. ${ }^{73}$ University of Southampton, Faculty of Medicine, Southampton, UK. ${ }^{74}$ Harvard T.H. Chan School of Public Health, Department of Epidemiology, Boston, MA, USA. ${ }^{75}$ Brigham and Women's Hospital and Harvard Medical School, Channing Division of Network Medicine, Boston, MA, USA. ${ }^{76}$ University of Leipzig, Institute for Medical Informatics, Statistics and Epidemiology, Leipzig, Germany. ${ }^{77}$ University of Leipzig, LIFE-Leipzig Research Centre for Civilization Diseases, Leipzig, Germany. ${ }^{78}$ University of Manchester, Manchester Academic Health Science Centre, Division of Evolution and Genomic Sciences, School of Biological Sciences, Faculty of Biology, Medicine and Health, Manchester, UK. ${ }^{79}$ St Mary's Hospital, Manchester University NHS Foundation Trust, Manchester Academic Health Science Centre, North West Genomics Laboratory Hub, Manchester Centre for Genomic Medicine, Manchester, UK. ${ }^{80}$ University of California at Los Angeles, David Geffen School of Medicine, Department of Medicine Division of Hematology and Oncology, Los Angeles, CA, USA. ${ }^{81}$ Imperial College London, Division of Cancer and Ovarian Cancer Action Research Centre, Department of Surgery and Cancer, London, UK. ${ }^{82}$ Masaryk Memorial Cancer Institute, Department of Cancer Epidemiology and Genetics, Brno, Czech Republic. ${ }^{83}$ Chaim Sheba Medical Center, The Susanne Levy Gertner Oncogenetics Unit, Ramat Gan, Israel. ${ }^{84}$ Tel Aviv University, Sackler Faculty of Medicine, Ramat Aviv, Israel. ${ }^{85}$ Jonsson Comprehensive Cancer Centre, UCLA, Schools of Medicine and Public Health, Division of Cancer Prevention \& Control Research, Los Angeles, CA, USA. ${ }^{86}$ Dana-Farber Cancer Institute, Cancer Risk and Prevention Clinic, Boston, 
MA, USA. ${ }^{87}$ University of Florence, Department of Experimental and Clinical Biomedical Sciences 'Mario Serio', Medical Genetics Unit, Florence, Italy. ${ }^{88}$ Cancer Council Victoria, Cancer Epidemiology Division, Melbourne, VIC, Australia. ${ }^{89}$ The University of Melbourne, Centre for Epidemiology and Biostatistics, Melbourne School of Population and Global Health, Melbourne, VIC, Australia. ${ }^{90}$ Monash University, Precision Medicine, School of Clinical Sciences at Monash Health, Clayton, VIC, Australia. ${ }^{91}$ University of Kansas Medical Center, Department of Pathology and Laboratory Medicine, Kansas City, KS, USA. ${ }^{92}$ Cedars-Sinai Medical Center, Samuel Oschin Comprehensive Cancer Institute, Cancer Prevention and Genetics Program, Los Angeles, CA, USA. ${ }^{93}$ National Cancer Institute, Clinical Genetics Branch, Division of Cancer Epidemiology and Genetics, Bethesda, MD, USA. ${ }^{94}$ Pomeranian Medical University, Department of Genetics and Pathology, Szczecin, Poland. ${ }^{95}$ QIMR Berghofer Medical Research Institute, Population Health Department, Brisbane, QLD, Australia. ${ }^{96}$ Faculty of Medicine and University Hospital Cologne, University of Cologne, Center for Integrated Oncology (ClO), Cologne, Germany. ${ }^{97}$ University of Southern California, Department of Preventive Medicine, Keck School of Medicine, Los Angeles, CA, USA. ${ }^{98}$ Karolinska Institutet, Institute of Environmental Medicine, Stockholm, Sweden. ${ }^{99}$ German Cancer Research Center (DKFZ), Molecular Genetics of Breast Cancer, Heidelberg, Germany. ${ }^{100}$ Rigshospitalet, Copenhagen University Hospital, Department of Clinical Genetics, Copenhagen, Denmark. ${ }^{101}$ Fred Hutchinson Cancer Research Center, Program in Epidemiology, Division of Public Health Sciences, Seattle, WA, USA. ${ }^{102}$ University of Washington, Department of Epidemiology, Seattle, WA, USA. ${ }^{103}$ National University of Singapore and National University Health System, Saw Swee Hock School of Public Health, Singapore, Singapore. ${ }^{104}$ National University Health System, Department of Surgery, Singapore, Singapore. ${ }^{105}$ Humboldt-Universität zu Berlin, and Berlin Institute of Health, Department for Gynecology with the Center for Oncologic Surgery Charité Campus Virchow-Klinikum, Charité - Universitätsmedizin Berlin, corporate member of Freie Universität Berlin, Berlin, Germany. ${ }^{106}$ University of Texas MD Anderson Cancer Center, Department of Epidemiology, Houston, TX, USA. ${ }^{107}$ Danish Cancer Society Research Center, Department of Virus, Lifestyle and Genes, Copenhagen, Denmark. ${ }^{108}$ University of Copenhagen, Molecular Unit, Department of Pathology, Herlev Hospital, Copenhagen, Denmark. ${ }^{109}$ University of Copenhagen, Department of Gynaecology, Rigshospitalet, Copenhagen, Denmark. ${ }^{110}$ Roswell Park Cancer Institute, Center For Immunotherapy, Buffalo, NY, USA. ${ }^{11}$ BC Cancer, Vancouver General Hospital, and University of British Columbia, British Columbia's Ovarian Cancer Research (OVCARE) Program, Vancouver, BC, Canada. ${ }^{112}$ University of British Columbia, Department of Pathology and Laboratory Medicine, Vancouver, BC, Canada. ${ }^{113}$ University of British Columbia, Department of Obstetrics and Gynecology, Vancouver, BC, Canada. ${ }^{114}$ BC Cancer Research Centre, Department of Molecular Oncology, Vancouver, BC, Canada. ${ }^{115}$ N.N. Petrov Institute of Oncology, St. Petersburg, Russia. ${ }^{116}$ Coordinating center: The Netherlands Cancer Institute, The Hereditary Breast and Ovarian Cancer Research Group Netherlands (HEBON), Amsterdam, The Netherlands. ${ }^{117}$ Lombardi Comprehensive Cancer Center, Georgetown University, Washington, DC, USA. ${ }^{118}$ Pomeranian Medical University, Independent Laboratory of Molecular Biology and Genetic Diagnostics, Szczecin, Poland. ${ }^{119}$ Peter MacCallum Cancer Center, Parkville Familial Cancer Centre, Melbourne, VIC, Australia. ${ }^{120}$ Vilnius University Hospital Santariskiu Clinics, Hematology, oncology and transfusion medicine center, Dept. of Molecular and Regenerative Medicine, Vilnius, Lithuania. ${ }^{121}$ State Research Institute Centre for Innovative Medicine, Vilnius, Lithuania. ${ }^{122}$ Landspitali University Hospital, Department of Oncology, Reykjavik, Iceland. ${ }^{123}$ Stanford University School of Medicine, Department of Epidemiology \& Population Health, Stanford, CA, USA. ${ }^{124}$ Stanford Cancer Institute, Stanford University School of Medicine, Department of Medicine, Division of Oncology, Stanford, CA, USA. ${ }^{125}$ The Institute of Cancer Research, Division of Genetics and Epidemiology, London, UK. ${ }^{126}$ Seoul National University College of Medicine, Department of Preventive Medicine, Seoul, Korea. ${ }^{127}$ Seoul National University Graduate School, Department of Biomedical Sciences, Seoul, Korea. ${ }^{128}$ Seoul National University, Cancer Research Institute, Seoul, Korea. ${ }^{129}$ University of California at Los Angeles, David Geffen School of Medicine, Department of Obstetrics and Gynecology, Los Angeles, CA, USA. ${ }^{130}$ UC Davis Medical Center, Department of Pathology and Laboratory Medicine, Sacramento, CA, USA. ${ }^{131}$ Medical University of South Carolina, Hollings Cancer Center, Charleston, SC, USA. ${ }^{132}$ Saint Petersburg State University, Saint Petersburg, Russia. ${ }^{133}$ Sungkyunkwan University School of Medicine, Department of Obstetrics and Gynecology, Samsung Medical Center, Seoul, Korea. ${ }^{134}$ City of Hope Clinical Cancer Genetics Community Research Network, Duarte, CA, USA. ${ }^{135}$ Cancer Genetics Centre, Hong Kong Hereditary Breast Cancer Family Registry, Happy Valley, Hong Kong. ${ }^{136}$ The University of Hong Kong, Department of Surgery, Pok Fu Lam, Hong Kong. ${ }^{137}$ Hong Kong Sanatorium and Hospital, Department of Surgery, Happy Valley, Hong Kong. ${ }^{138} \mathrm{VIB}$ Center for Cancer Biology, Leuven, Belgium. ${ }^{139}$ University of Leuven, Laboratory for Translational Genetics, Department of Human Genetics, Leuven, Belgium. ${ }^{140}$ Mayo Clinic, Department of Health Sciences Research, Division of Biomedical Statistics and Informatics, Rochester, MN, USA. ${ }^{141}$ ONCOBELL-IDIBELLIGTP, Catalan Institute of Oncology, CIBERONC, Hereditary Cancer Program, Barcelona, Spain. ${ }^{142}$ BC Cancer, Cancer Control Research, Vancouver, BC, Canada. ${ }^{143}$ Inserm U900, Genetic Epidemiology of Cancer team, Paris, France. ${ }^{144}$ NYU Langone Medical Center, Gynecologic Oncology, Laura and Isaac Pearlmutter Cancer Center, New York, NY, USA. ${ }^{145}$ Genome Institute of Singapore, Human Genetics Division, Singapore, Singapore. ${ }^{146}$ University of Texas MD Anderson Cancer Center, Department of Gynecologic Oncology and Clinical Cancer Genetics Program, Houston, TX, USA. ${ }^{147}$ Magee-Womens Hospital, University of Pittsburgh School of Medicine, Pittsburgh, PA, USA. ${ }^{148}$ Fondazione IRCCS Istituto Nazionale dei Tumori di Milano, Unit of Medical Genetics, Department of Medical Oncology and Hematology, Milan, Italy. ${ }^{149}$ Duke University Hospital, Department of Surgery, Durham, NC, USA. ${ }^{150}$ University of Hawaii Cancer Center, Cancer Epidemiology Program, Honolulu, HI, USA. ${ }^{151}$ Aichi Cancer Center Research Institute, Division of Cancer Epidemiology and Prevention, Nagoya, Japan. ${ }^{152}$ Nagoya University Graduate School of Medicine, Division of Cancer Epidemiology, Nagoya, Japan. ${ }^{153}$ Samuel Lunenfeld Research Institute, Public Health Ontario, Toronto, ON, Canada. ${ }^{154}$ Imperial College London, Division of Cancer and Ovarian Cancer Action Research Centre, Department Surgery \& Cancer, London, UK. ${ }^{155}$ University of Glasgow, Institute of Cancer Sciences, Glasgow, UK. ${ }^{156}$ University College London, MRC Clinical Trials Unit at UCL, Institute of Clinical Trials \& Methodology, London, UK. ${ }^{157}$ Roswell Park Cancer Institute, NRG Oncology, Statistics and Data Management Center, Buffalo, NY, USA. ${ }^{158}$ Roswell Park Cancer Institute, Division of Cancer Prevention and Control, Buffalo, NY, USA. ${ }^{159}$ Magee-Womens Research Institute and Hillman Cancer Center, Womens Cancer Research Center, Pittsburgh, PA, USA. ${ }^{160}$ University of Pittsburgh School of Medicine, Division of Gynecologic Oncology, Department of Obstetrics, Gynecology and Reproductive Sciences, Pittsburgh, PA, USA. ${ }^{161}$ Oregon Health \& Science University, Department of Obstetrics and Gynecology, Portland, OR, USA. ${ }^{162}$ Oregon Health \& Science University, Knight Cancer Institute, Portland, OR, USA. ${ }^{163}$ University of Helsinki, Department of Obstetrics and Gynecology, Helsinki University Hospital, Helsinki, Finland. ${ }^{164}$ National Cancer Centre, Cancer Genetics Service, Singapore, Singapore. ${ }^{165}$ Nanyang Technological University, Lee Kong Chian School of Medicine, Singapore, Singapore. ${ }^{166}$ Odense University Hospital, Department of Clinical Genetics, Odence C, Denmark. ${ }^{167}$ Latvian Biomedical Research and Study Centre, Riga, Latvia. ${ }^{168}$ Roswell Park Cancer Institute, Department of Gynecologic Oncology, Buffalo, NY, USA. ${ }^{169}$ Memorial Sloan Kettering Cancer Center, Clinical Genetics Research Lab, Department of Cancer Biology and Genetics, New York, NY, USA. ${ }^{170}$ Memorial Sloan Kettering Cancer Center, Clinical Genetics Service, Department of Medicine, New York, NY, USA. ${ }^{171}$ National Institute of Oncology, Department of Molecular Genetics, Budapest, Hungary. ${ }^{172}$ University Hospitals Leuven, Division of Gynecologic Oncology, Department of Obstetrics and Gynaecology and Leuven Cancer Institute, Leuven, Belgium. ${ }^{173}$ The University of Chicago, Center for Clinical Cancer Genetics, Chicago, IL, USA. ${ }^{174}$ Memorial Sloan-Kettering Cancer Center, Department of Epidemiology and Biostatistics, New York, NY, USA. ${ }^{175}$ Centro de Investigación en Red de Enfermedades Raras (CIBERER), Madrid, Spain. ${ }^{176}$ QIMR Berghofer Medical Research Institute, Department of Genetics and Computational Biology, Brisbane, QLD, Australia. ${ }^{177}$ Aalborg University Hospital, Molecular Diagnostics, Aalborg, Denmark. ${ }^{178}$ Aalborg University Hospital, Clinical Cancer Research Center, Aalborg, Denmark. ${ }^{179}$ Aalborg University, Department of Clinical Medicine, Aalborg, Denmark. ${ }^{180}$ Portuguese Oncology Institute, Department of Genetics, Porto, Portugal. ${ }^{181}$ Moffitt Cancer Center, Department of Cancer Epidemiology, Tampa, FL, USA. ${ }^{182}$ IFOM - the FIRC Institute of Molecular Oncology, Genome Diagnostics Program, Milan, Italy. ${ }^{183}$ Bashkir State University, Department of Genetics and Fundamental Medicine, Ufa, Russia. ${ }^{184}$ Fondazione IRCCS Istituto Nazionale dei Tumori (INT), Unit of Molecular Bases of Genetic Risk and Genetic Testing, Department of Research, Milan, Italy. ${ }^{185}$ Karolinska Institutet, Clinical Genetics, Stockholm, Sweden. ${ }^{186}$ Duke University Hospital, Department of Gynecologic Oncology, Durham, NC, USA. ${ }^{187}$ Yale School of Public Health, Chronic Disease Epidemiology, New Haven, CT, USA. ${ }^{188}$ Fox Chase Cancer Center, Population Studies Facility, Philadelphia, PA, USA. ${ }^{189}$ National Institute of Environmental Health Sciences, NIH, Epidemiology Branch, Research Triangle Park, NC, USA. ${ }^{190}$ Fundación Pública Galega Medicina Xenómica, Santiago De Compostela, Spain. ${ }^{191}$ Instituto de Investigación Sanitaria de Santiago de Compostela, Santiago De Compostela, Spain. ${ }^{192}$ Centre Hospitalier Universitaire de Québec - Université Laval Research Center, Genomics Center, Québec City, QC, Canada. ${ }^{193}$ Faculty of Medicine and University Hospital Cologne, University of Cologne, Center for Molecular Medicine Cologne (CMMC), Cologne, Germany. ${ }^{194}$ Hebei Medical University, Fourth Hospital, Department of Obstetrics and Gynaecology, Shijiazhuang, China. ${ }^{195} \mathrm{Icahn}$ School of Medicine at Mount Sinai, Department of Population Health Science and Policy, New York, NY, USA. ${ }^{196}$ Icahn School of Medicine at Mount Sinai, Department of Genetics and Genomic Sciences, New York, NY, USA. ${ }^{197}$ Centre Hospitalier Universitaire de Québec-Université Laval Research Center, Genomic Center, Québec City, QC, Canada. ${ }^{198}$ Medical University of Vienna, Dept of OB/GYN and Comprehensive Cancer Center, Vienna, Austria. ${ }^{199}$ University of Cambridge, Department of Public Health and Primary Care, Cambridge, UK. ${ }^{200}$ The University of Melbourne, Department of Clinical Pathology, Melbourne, VIC, Australia. ${ }^{201}$ Royal Alexandra Hospital, Department of Obstetrics and Gynecology, Division of Gynecologic Oncology, Edmonton, AB, Canada. ${ }^{202}$ Institut Curie, Service de Génétique, Paris, France. ${ }^{203}$ Université Paris Descartes, Paris, France. ${ }^{204}$ University of South Florida, Epidemiology Center, College of Medicine, Tampa, FL, USA. ${ }^{205}$ The Institute of Cancer Research, Division of Breast Cancer Research, London, UK. ${ }^{206}$ University of Porto, Biomedical Sciences Institute (ICBAS), Porto, Portugal. ${ }^{207}$ Cancer Research Malaysia, Breast Cancer Research Programme, Subang Jaya, Selangor, Malaysia. ${ }^{208}$ University of Malaya, Department of Surgery, Faculty of Medicine, Kuala Lumpur, Malaysia. ${ }^{209}$ Brigham and Women's Hospital and Harvard Medical School, Obstetrics and Gynecology Epidemiology Center, Boston, MA, USA. ${ }^{210}$ Columbia University, Department of Epidemiology, Mailman School of Public Health, New York, NY, USA. ${ }^{211}$ MageeWomens Hospital, University of Pittsburgh School of Medicine, Department of Medicine, Pittsburgh, PA, USA. ${ }^{212}$ McGill University, Program in Cancer Genetics, Departments of 
Human Genetics and Oncology, Montréal, QC, Canada. ${ }^{213}$ University of Cambridge, Department of Medical Genetics, Cambridge, UK. ${ }^{214}$ Dartmouth College, Geisel School of Medicine, Hanover, NH, USA. ${ }^{215}$ The Ohio State University, Department of Cancer Biology and Genetics, Columbus, OH, USA. ${ }^{216}$ Pontificia Universidad Javeriana, Institute of Human Genetics, Bogota, Colombia. ${ }^{217}$ University of Oxford, Cancer Epidemiology Unit, Oxford, UK. ${ }^{218}$ Beth Israel Deaconess Medical Center, Department of Medical Oncology, Boston, MA, USA. ${ }^{219}$ University Medical Center Groningen, University Groningen, Department of Genetics, Groningen, The Netherlands. ${ }^{220}$ University of Pretoria, Department of Genetics, Arcadia, South Africa. ${ }^{221}$ Fundación Pública Galega de Medicina Xenómica, Santiago de Compostela, Spain. ${ }^{222}$ Instituto de Investigación Sanitaria de Santiago de Compostela (IDIS), Complejo Hospitalario Universitario de Santiago, SERGAS, Santiago de Compostela, Spain. ${ }^{223}$ Vanderbilt University Medical Center, Division of Quantitative Sciences, Department of Obstetrics and Gynecology, Department of Biomedical Sciences, Women's Health Research, Nashville, TN, USA. ${ }^{224}$ Duke Cancer Institute, Cancer Control

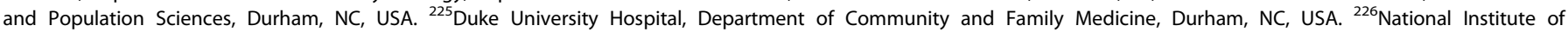
Environmental Health Sciences, NIH, Biostatistics and Computational Biology Branch, Research Triangle Park, NC, USA. ${ }^{227}$ City of Hope, Clinical Cancer Genomics, Duarte, CA, USA.

${ }^{228}$ Fred Hutchinson Cancer Research Center, Seattle, WA, USA. ${ }^{229}$ Stanford University School of Medicine, Department of Biomedical Data Science, Stanford, CA, USA. ${ }^{230}$ Uppsala University, Department of Surgical Sciences, Uppsala, Sweden. ${ }^{231}$ University of Malaya, Department of Obstetrics and Gynaecology, University of Malaya Medical Centre, Kuala Lumpur, Malaysia. ${ }^{232}$ Hebei Medical University, Fourth Hospital, Department of Molecular Biology, Shijiazhuang, China. ${ }^{233}$ National Centre for Scientific Research 'Demokritos', Molecular Diagnostics Laboratory, INRASTES, Athens, Greece. ${ }^{234}$ Institute of Biochemistry and Experimental Oncology, First Faculty od Medicine, Charles University, Prague, Czech Republic. ${ }^{235}$ Women's Cancer Program at the Samuel Oschin Comprehensive Cancer Institute, Cedars-Sinai Medical Centre, Department of Obstetrics and Gynecology, Los Angeles, CA, USA. ${ }^{236}$ Royal Pass Road, Tampa, FL, USA. ${ }^{237}$ University of NSW Sydney, School of Women's and Children's Health, Faculty of Medicine, Sydney, NSW, Australia. ${ }^{238}$ University of NSW Sydney, Adult Cancer Program, Lowy Cancer Research Centre, Sydney, NSW, Australia. ${ }^{239}$ University of Michigan School of Public Health, Department of Epidemiology, Ann Arbor, MI, USA. ${ }^{240}$ University of Southern California Norris Comprehensive Cancer Center, Department of Preventive Medicine, Keck School of Medicine, Los

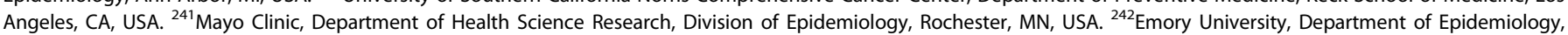
Rollins School of Public Health, Atlanta, GA, USA. ${ }^{243}$ These authors contributed equally: Eileen O. Dareng, Jonathan P. Tyrer. ${ }$ email: pp10001@medschl.cam.ac.uk

\section{GEMO Study Collaborators}

Fabienne Lesueur and Noura Mebirouk

GC-HBOC Study Collaborators

Christoph Engel and Rita K. Schmutzler

\section{EMBRACE Collaborators}

Daniel Barrowdale, Eleanor Davies, Diana M. Eccles and D. Gareth Evans

\section{KConFab Investigators}

Georgia Chenevix-Trench

\section{HEBON Investigators}

Muriel A. Adank, Peter Devilee and Annemieke H. van der Hout

\section{The OCAC Consortium}

Eileen O. Dareng, Jonathan P. Tyrer, Michelle R. Jones, Katja K. H. Aben, Hoda Anton-Culver, Natalia N. Antonenkova, Gerasimos Aravantinos, Matthias W. Beckmann, Alicia Beeghly-Fadiel, Javier Benitez, Marina Bermisheva, Marcus Q. Bernardini, Line Bjorge, Natalia V. Bogdanova, James D. Brenton, Agnieszka Budzilowska, Ralf Butzow, Hui Cai, lan Campbell, Rikki Cannioto, Jenny Chang-Claude, Stephen J. Chanock, Kexin Chen, Yoke-Eng Chiew, Linda S. Cook, Fanny Dao, Joe Dennis, Jennifer A. Doherty, Thilo Dörk, Andreas du Bois, Matthias Dürst, Diana M. Eccles, Heather A. Eliassen, Peter A. Fasching, James M. Flanagan, Renée T. Fortner, Graham G. Giles, Marc T. Goodman, Jacek Gronwald, Christopher A. Haiman, Niclas Håkansson, Holly R. Harris, Florian Heitz, Michelle A. T. Hildebrandt, Estrid Høgdall, Claus K. Høgdall, Ruea-Yea Huang, Chad Huff, David G. Huntsman, Anna Jakubowska, Allan Jensen, Michael E. Jones, Daehee Kang, Beth Y. Karlan, Anthony Karnezis, Linda E. Kelemen, Elza Khusnutdinova, Lambertus A. Kiemeney, Byoung-Gie Kim, Susanne K. Kjaer, Jolanta Kupryjanczyk, Diether Lambrechts, Melissa C. Larson, Nhu D. Le, Jenny Lester, Douglas A. Levine, Karen H. Lu, Jan Lubiński, Jeffrey R. Marks, Rayna Kim Matsuno, Keitaro Matsuo, Taymaa May, John R. McLaughlin, lain A. McNeish, Roger L. Milne, Albina Minlikeeva, Francesmary Modugno, Kirsten B. Moysich, Elizabeth Munro, Heli Nevanlinna, Kunle Odunsi, Siel Olbrecht, Sara H. Olson, Håkan Olsson, Ana Osorio, Sue K. Park, Tanja Pejovic, Jennifer B. Permuth, Anna Piskorz, Darya Prokofyeva, Marjorie J. Riggan, Harvey A. Risch, Cristina Rodriguez-Antona, Mary Anne Rossing, Ingo Runnebaum, Dale P. Sandler, V. Wendy Setiawan, Kang Shan, Weiva Sieh, Honglin Song, Melissa C. Southey, Helen Steed, Rebecca Sutphen, Anthony J. Swerdlow, Soo Hwang Teo, Kathryn L. Terry, Pamela J. Thompson, Liv Cecilie Vestrheim Thomsen, Linda Titus, Britton Trabert, Ruth Travis, Shelley S. Tworoger, Ellen Valen, Anne M. van Altena, Els Van Nieuwenhuysen, Digna Velez Edwards, Robert A. Vierkant, Frances Wang, Penelope M. Webb, Clarice R. Weinberg, Nicolas Wentzensen, Emily White, Alice S. Whittemore, Stacey J. Winham, Alicja Wolk, Yin-Ling Woo, Anna H. Wu, Li Yan, Drakoulis Yannoukakos, Wei Zheng, Argyrios Ziogas, Kate Lawrenson, Anna deFazio, Susan J. Ramus, Celeste L. Pearce, Alvaro N. Monteiro, Julie M. Cunningham, Ellen L. Goode, Joellen M. Schildkraut, Andrew Berchuck, Simon A. Gayther and Paul D. P. Pharoah 


\section{The CIMBA Consortium}

Daniel R. Barnes, Xin Yang, Muriel A. Adank, Simona Agata, Irene L. Andrulis, Banu K. Arun, Annelie Augustinsson, Judith Balmaña, Rosa B. Barkardottir, Daniel Barrowdale, Bernardo Bonanni, Ake Borg, Saundra S. Buys, Maria A. Caligo, Hayley Cassingham, Wendy K. Chung, Kathleen B. M. Claes, Sarah Colonna, Fergus J. Couch, Mary B. Daly, Eleanor Davies, Miguel de la Hoya, Robin de Putter, Allison DePersia, Peter Devilee, Orland Diez, Yuan Chun Ding, Susan M. Domchek, Diana M. Eccles, Christoph Engel, D. Gareth Evans, Eva Machackova, Eitan Friedman, Patricia A. Ganz, Judy Garber, Francesca Gensini, Gord Glendon, Andrew K. Godwin, Mark H. Greene, Eric Hahnen, Ute Hamann, Thomas V. O. Hansen, Mikael Hartman, John L. Hopper, Peter J. Hulick, Evgeny N. Imyanitov, Claudine Isaacs, Paul A. James, Ramunas Janavicius, Oskar Th. Johannsson, Esther M. John, lan Komenaka, Allison W. Kurian, Ava Kwong, Conxi Lazaro, Goska Leslie, Fabienne Lesueur, Jingmei Li, Jennifer T. Loud, Phuong L. Mai, Siranoush Manoukian, Lesley McGuffog, Noura Mebirouk, Austin Miller, Marco Montagna, Katherine L. Nathanson, Susan L. Neuhausen, Joanne Ngeow Yuen Yie, Henriette Roed Nielsen, Liene Nikitina-Zake, Kenneth Offit, Edith Olah, Olufunmilayo I. Olopade, Laura Papi, Michael T. Parsons, Harsha Pathak, Inge Sokilde Pedersen, Ana Peixoto, Pedro Perez-Segura, Beth Peshkin, Paolo Peterlongo, Paolo Radice, Johanna Rantala, Eric Ross, Marta Santamariña, Penny Soucy, Rita K. Schmutzler, Jacques Simard, Christian F. Singer, Anna P. Sokolenko, Dominique Stoppa-Lyonnet, Yen Yen Tan, Manuel R. Teixeira, Mary Beth Terry, Mads Thomassen, Darcy L. Thull, Marc Tischkowitz, Amanda E. Toland, Diana Torres, Nadine Tung, Annemieke H. van der Hout, Elizabeth J. van Rensburg, Ana Vega, Barbara Wappenschmidt, Jeffrey N. Weitzel, Katia M. Zavaglia, Kristin K. Zorn, Thomas A. Sellers, Georgia Chenevix-Trench and Antonis C. Antoniou 